\title{
A Constrained Mixture Theory Model to Study Autoregulation in the Coronary Circulation
}

\author{
Hamidreza Gharahi ${ }^{1}$, Daniel A. Beard ${ }^{2}$, C. Alberto Figueroa ${ }^{1,3^{*}}$, Seungik Baek ${ }^{4^{*}}$ \\ ${ }^{1}$ Section of Vascular Surgery, Department of Surgery, University of Michigan, Ann Arbor, MI 48109, USA \\ ${ }^{2}$ Department of Molecular and Integrative Physiology, University of Michigan, Ann Arbor, Michigan 48109, USA \\ ${ }^{3}$ Departments Biomedical Engineering, University of Michigan, Ann Arbor, MI 48109, USA \\ ${ }^{4}$ Department of Mechanical Engineering, Michigan State University, East Lansing, MI 48824, USA
}

\section{Key points}

- Coronary autoregulation is defined as the capability of the coronary circulation to maintain the blood supply to the heart over a range of perfusion pressures. This phenomenon is facilitated through intrinsic mechanisms that control the vascular resistance by regulating the mechanical function of smooth muscle cells. Understanding the mechanisms involved in coronary autoregulation is one of the most fundamental questions in coronary physiology.

- This paper presents a structurally-motivated coronary autoregulation model that uses a nonlinear continuum mechanics approach to account for the morphometry and vessel wall composition in two coronary trees in the subepicardial and subendocardial layers.

- The model is calibrated against diverse experimental data from literature and is used to study heterogeneous autoregulatory response in the coronary trees. This model drastically differs from previous models, which relied on lumped parameter model formulations, and is suited to the study of long-term pathophysiological growth and remodeling phenomena in coronary vessels.

\begin{abstract}
Coronary autoregulation is a short-term response manifested by a relatively constant flow over a wide range of perfusion pressures for a given metabolic state. This phenomenon is thought to be facilitated through a combination of mechanisms, including myogenic, shear dependent, and metabolic controls. The study of coronary autoregulation is challenging due to the coupled nature of the mechanisms and their differential effects through the coronary tree. In this paper, we developed a novel framework to study coronary autoregulation based on the constrained mixture theory. This structurally-motivated autoregulation model required calibration of anatomical and structural parameters of coronary trees via a homeostatic optimization approach using extensive literature data. Autoregulation was then simulated for two different coronary trees: subepicardial and subendocardial. The structurally calibrated model reproduced available baseline hemodynamics and autoregulation data for each coronary tree. The autoregulation analysis showed that the diameter of the intermediate and small arterioles varies the most in response to changes in perfusion pressure. Finally, we demonstrated the utility of the model in two application examples: 1) response to drops in epicardial pressure, and 2) response to drug infusion in the coronary arteries. The proposed structurally-motivated model could be extended to study long-term growth and remodeling in the coronary circulation in response to hypertension, atherosclerosis, etc.
\end{abstract}

\footnotetext{
*Corresponding authors. Email addresses figueroc@med.umich.edu (C. Alberto Figueroa), sbaek@egr.msu.edu (Seungik Baek)
} 


\section{Introduction}

Coronary blood flow rapidly changes to meet changes in metabolic demand (myocardial oxygen consumption, $\mathrm{MVO}_{2}$ ) (25). This coronary flow regulation is the result of vessel diameter modulation via local control of the active stress in the smooth muscle cells (SMCs) (31). One manifestation of coronary flow regulation is the so-called pressure-flow autoregulation, in which perturbations in coronary perfusion pressure are met with commensurate changes in microvascular resistance to maintain a constant coronary blood flow at a given level of $\mathrm{MVO}_{2}(20)$.

Our current understanding of the different control mechanisms involved in coronary autoregulation is rather phenomenological. Briefly, coronary autoregulation relies on SMCs reactivity regulated through myogenic, shear-dependent, and metabolic control mechanisms. Myogenic control is the intrinsic response of SMCs to changes in the local wall stress (52). Shear-dependent control is a dilatory mechanism mediated via shearinduced production of nitric oxide (NO) by the endothelial cells. Metabolic control is a local feedback mechanism where a vasodilation signal is initiated in response to either an increase in local oxygen consumption or oxygen extraction from the myocardial blood supply (25). Studies have shown that these mechanisms heterogeneously affect the coronary microvasculature (81). Myogenic control has been observed to be small in precapillary arterioles and large in the arteries of 50-150 $\mu \mathrm{m}$ (55). Shear-dependent control is most active in the arteries and larger arterioles (54). Lastly, the signal for the metabolic feedback mechanism is initiated in the capillaries, conducted upstream, and it gradually decays for larger vessels $(\sim 150 \mu \mathrm{m})$ (44). Furthermore, coronary autoregulation occurs across different layers of the myocardium where the myocardial-vessel interactions are not uniform (92). Therefore, the anatomical and functional heterogeneity of the coronary arteries, and the difficulties in acquiring in-vivo measurements necessitate the development of computational models that can integrate the available experimental data on morphometry, biomechanical, and physiological responses to enhance our understanding of coronary autoregulation in healthy, disease, and treatment planning conditions (e.g., coronary artery disease, cardiac hypertrophy, metabolic dysfunction, etc.) (23).

Several computational techniques have been used to model the morphometry and hemodynamics of coronary arteries $(1,12,39,46,77)$. Kaimovitz et al. $(42,43)$ provided a stochastic framework for constructing the entire coronary arterial vasculature using a volume-filling optimization subject to morphometric statistics reported in (48). Namani et al. (67) extended Kaimovitz's model by incorporating physiological hemodynamic constraints in the generation of the coronary arterial network. They showed that a concurrent minimization of flow dispersion and diameter re-assignment at the microvascular level resulted in a realistic representation of the network when compared with literature data. These coronary arterial tree reconstruction methods, while informed by morphometry and hemodynamic data, have not considered structurally-motivated data for the passive and active behavior of the coronary vessels at the different generations of the network.

On the other hand, the phenomenon of coronary autoregulation has been the subject of numerous modeling studies over the last 50-60 years. Virtually all of such studies have relied on lumped-parameter (0D) approaches. Liao and Kuo (55) and Cornelissen et al. (18) investigated the interaction and balance between autoregulatory mechanisms in the coronary circulation. While these studies incorporated the heterogeneity of the microvascular response, they considered a simple partitioning of the coronary tree into 4 compartments and ignored the interactions between vessels and myocardium. Pradhan et al. (69) applied a data-driven closed loop model of coronary flow regulation using in-vivo data on coronary flow and oxygen extraction in response to exercise-induced changes in demand and to changes in perfusion pressure. The Pradhan model, which represents parallel control pathways using a block-diagram approach, does not 
represent explicit spatial features. More recently, Namani and colleagues (66) developed a model of the coronary microcirculation that integrated the dynamic effects of coronary flow with myogenic, shear dependent, and metabolic feedback control mechanisms in subendocardial and subepicardial vessels. Their constitutive model considered simple pressure-diameter rules to define the behavior of the vessels. While the aforementioned studies provide reasonable descriptions of autoregulatory responses, they lacked structurally-motivated models for arterial tissue. Given that the short-term regulation of vascular tone and the long-term vascular growth and remodeling processing, including pathophysiologic responses, are highly intertwined (85), there is a pressing need to develop models of coronary autoregulation that can account for the microstructure of the vessel wall.

Constrained mixture theory models have been widely applied to describe the nonlinear mechanical behavior of arterial tissue, accounting for the contributions of the main load-bearing constituents (e.g., collagen, elastin and SMCs) $(26,74)$. This theory provides a formal means to represent mechanical function of a vessel based on the properties of its constituents and the relative mass fractions of those constituents in the composition of the vessel wall. Using this theory, long-term growth and remodeling processes are represented by changes in the composition of the vessel wall. Previous application of the constrained mixture theory in vascular mechanics have focused on large arteries where homeostatic stress has been assumed constant. However, wall stress has been found to be size-dependent through the vasculature (33, 71). Motivated by this, Filonova and colleagues (29) developed a homeostatic optimization framework, adopted from an extended Murray law $(56,80)$, to generate vascular trees obeying a data-based fractal, to predict homeostatic characteristics, such as morphometry, hemodynamics, and structure.

The goal of this study is to apply the homeostatic optimization framework of Filonova et al. to develop a structurally-motivated computational model of coronary autoregulation, a physiological mechanism that fundamentally relies on mechanical function of SMCs. The model, based on the constrained mixture theory, provides a means to estimates baseline activation of SMCs and homeostatic stresses, while being informed by experimental pressure-diameter, tree morphometry, and hemodynamics data, for both subepicardial and subendocardial trees. In this work, our model is limited to calculating the steady-state response to stimuli triggering combined alterations in metabolic, shear-dependent, and myogenic mechanisms. Lastly, the utility of our model is demonstrated using two application examples: 1) study of short-term adaptations following drops in epicardial pressure (Section 3.4), and 2) simulation of the short-term coronary responses to pharmacological agents such as adenosine (which binds to purinergic receptors and causes SMC relaxation) and L-NAME infusion (which inhibits NO synthesis and therefore induces vasoconstriction) (Section 3.5). An additional benefit of our approach is that this model could also be extended to study longterm growth and remodeling in the coronary circulation in response to hypertension, atherosclerosis, etc., thereby rendering a unified framework to describe the dynamics of both short- and long-term coronary vascular adaptations.

\section{Methods}

\subsection{Model overview and parameter estimation process}

Coronary arterial networks embedded in the subendo- and subepicardial layers are represented as bifurcating trees. A steady state fluid model describes the relation between blood flow and pressure through estimation of vascular resistance in each idealized tree segment. The geometry and composition of vessel segments at each generation of the tree are defined based on minimization of energy consumption and the mechanical equilibrium which relate the diameter, pressure, and vessel wall thickness. The model uses a nonlinear continuum mechanics approach and is endowed with autoregulatory mechanisms to study the 
coronary autoregulation. Overall, our model determines the diameter of each vessel segment as a function of the coronary perfusion pressure by integrating the hemodynamics of the tree and autoregulatory responses.

The strategy to calibrate the model is summarized in Fig. 1. The first stage is to estimate the constrained mixture model parameters, representing the material properties of load-bearing constituents within the vessel wall (elastin, collagen, SMCs), their respective pre-stretches (the stretch at which the constituents are deposited in homeostatic conditions), and their mass fractions, for four vessel types (small arteries, large, intermediate, and small arterioles), for both the subepicardium and subendocardium layers. These parameters are estimated by comparing model predictions to data on measurements of the passive and full myogenic response of isolated porcine coronary vessels to internal pressurization (55). In addition to the constrained mixture model parameters, this step also identifies model parameters associated with the myogenic component of the autoregulatory response.

Second, using the homeostatic optimization framework proposed by Filonova and colleagues (29), we generate two coronary trees in the subendocardial and subepicardial layers and establish their homeostatic characteristics, i.e., morphometry, hemodynamics, and structure. Motivated by Murray's law (63), the homeostatic optimization framework defines the homeostatic state (equilibrium) of a vascular tree to be governed by an energy-based minimization problem in which the energy dissipation includes the power needed to overcome viscous drag and the metabolic energy spent on sustaining blood supply and maintaining the constituents of the vascular wall. This minimization is performed under mechanical equilibrium as a constraint.

Third, a structurally-motivated coronary autoregulation model was developed, integrating the myogenic with metabolic and shear-dependent mechanisms, for each of the two coronary trees. In the following sections, we describe each of the three stages in detail, together with the experimental data they require.

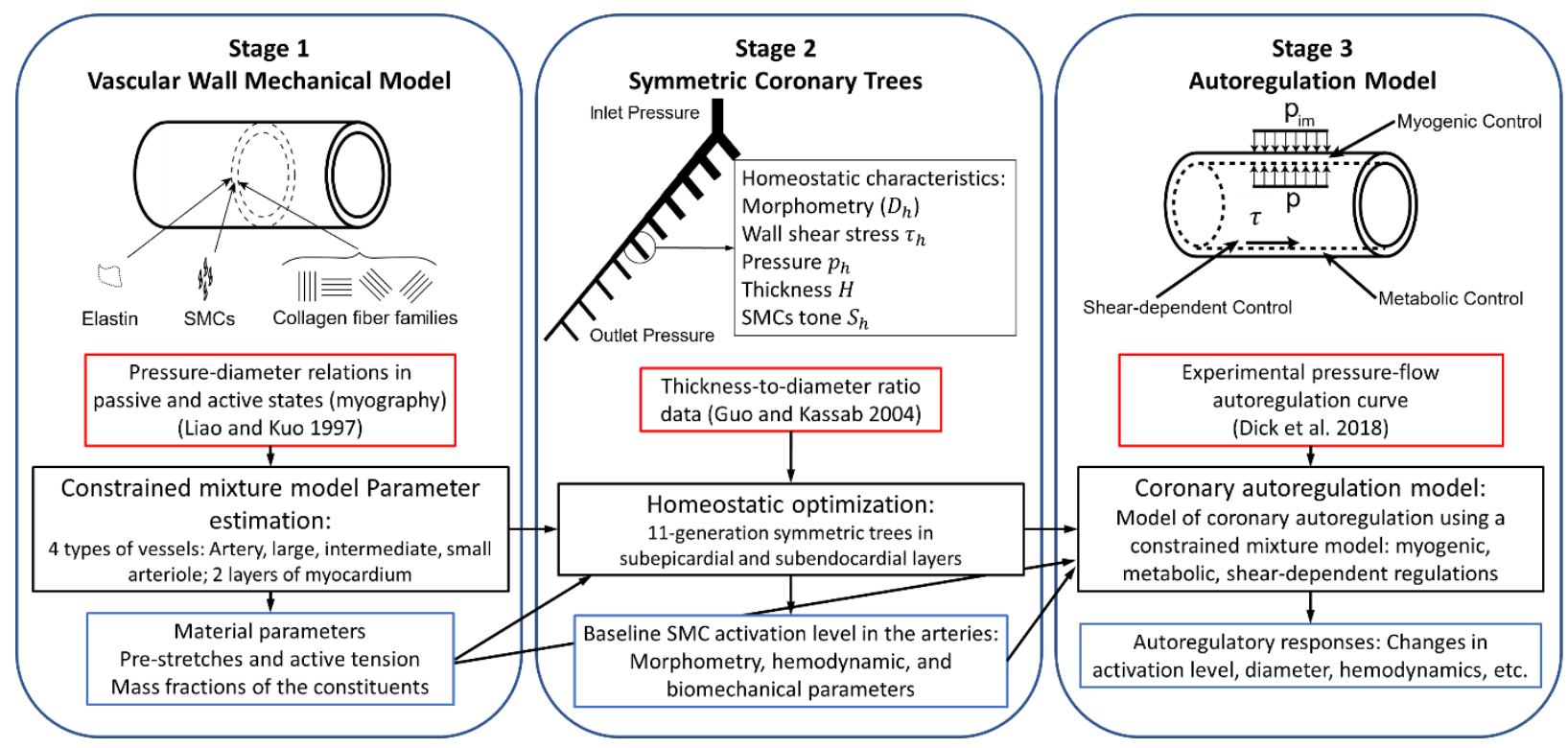

Figure 1. Schematic of the components and the workflow of modeling stages and data in the proposed constrained mixture theory model of coronary autoregulation. The arrows show the flow of information. Red, black, and blue boxes delineate the literature data, the methods used, and model results, respectively. 


\subsection{Parameter estimation for the constrained mixture model of 8 representative coronary vessels}

A constrained mixture theory model is used to describe the arterial tissue mechanics with three main loadbearing constituents: elastin matrix, collagen fibers, and SMCs. This approach incorporates microstructural properties and cellular level functions of the vessel within a nonlinear continuum mechanics approach, whereby the wall constituents are constrained to deform together but have mechanical properties and stresses. The relationship between transmural pressure $p_{t m}$ and internal diameter $D$ in a thin walled cylinder is given by Laplace's law: $p_{t m} D / 2=T_{\theta \theta}$, where the circumferential tension $T_{\theta \theta}$ is determined by contributions from elastin, collagen and SMCs. Each constituent has distinct strain energy densities $(w)$ mapping deformation gradients from their distinct stress-free configurations to the overall in-vivo configuration. In this work, we considered a neo-Hookean model for elastin (with coefficient $c_{1}$ ) and a Holzapfel's exponential model for i) collagen fiber families (with coefficients $c_{2}$ and $c_{3}$ ) and ii) passive response of SMCs $\left(c_{4}\right.$ and $\left.c_{5}\right)$. The active tension $\left(T_{a c t}\right)$ exerted by SMCs, was defined as

$$
T_{\text {act }}=\frac{M_{R}^{m}}{\rho_{\text {wall }}} S\left(1-\left(\frac{\lambda_{M}-\lambda_{\theta}}{\lambda_{M}-\lambda_{0}}\right)^{2}\right),
$$

where $S$ is the active SMC stress, $\rho_{\text {wall }}$ is the density of arterial tissue, $M_{R}^{m}$ areal density of SMCs, $\lambda_{M}$ and $\lambda_{0}$ are stretches corresponding to the maximum and zero active tension, respectively, and $\lambda_{\theta}$ is the vessel circumferential stretch. Since details of general mathematical forms of passive and active behaviors of the constrained mixture theory models are available elsewhere (10), they are briefly summarized in Appendix A.

The three main control mechanisms controlling active SMC stress $S$ in coronary autoregulation are myogenic, shear-dependent, and metabolic. In this work, we are interested in a formulation that separates the myogenic contribution from the shear-dependent and metabolic contributions to SMC stress $S$. Therefore, we have assumed the following functional relationship for $S$ :

$$
S=A\left(\tau, M V O_{2}\right) \times S_{p}\left(p_{t m}\right),
$$

where $A$ is the activation level $(0<A<1)$, determined by wall shear stress $(\tau)$ and metabolic activity $\left(\mathrm{MVO}_{2}\right)$ mechanisms; and $S_{p}$ is a pressure-dependent stress representing the level of myogenic control. Thus, when $A=0$, a given vessel is in a maximally dilated state, regardless of any influence of the myogenic mechanism. When $A=1$, there is no vasodilation, and the control of smooth muscle activation is exclusively through the myogenic mechanism. Following Cornelissen and colleagues (18), we assume that $S_{p}$ follows a Hill curve for positive transmural pressures and is zero in negative transmural pressures:

$$
\begin{gathered}
S_{p}\left(p_{t m}\right)=S_{\max } \frac{p_{t m}^{\beta}}{p_{t m}^{\beta}+p_{0}^{\beta}} \text { for } p_{t m} \geq 0, \text { and } \\
S_{p}\left(p_{t m}\right)=0 \text { for } p_{t m}<0,
\end{gathered}
$$

where $\beta$ is the Hill slope, $S_{\max }$ is the total maximum active stress, and $p_{0}$ is a parameter that offsets the center of the curve.

In addition to the material parameters determining the active and passive behavior of individual constituents, other important parameters that define the mechanical homeostatic condition in the constrained mixture models of vascular tissue are: 1) the homeostatic pre-stretches at which each 
constituent is deposited ( $G_{\theta}^{e}, G_{z}^{e}, G_{h}^{c}$, and $G_{h}^{m}$, see Appendix A for details), and 2) the fraction of the vessel wall occupied by each constituent (mass fractions $v^{e}, v^{c}$, and $v^{m} ; v^{e}+v^{c}+v^{m}=1$ ).

Constrained mixture models have been widely used in the computational study of growth and remodeling of large arteries, under the general assumption that the homeostatic stress of each wall constituent is constant $(2,28,30,91)$. However, the homeostatic stress of each constituent is unlikely to remain constant along the tree, since stresses such as hoop stress and wall shear stress vary significantly from large arteries to small arterioles $(29,71)$. Therefore, to account for this likely variability in constituent homeostatic stress, in this work we assumed that the mechanical properties of constituents (material stiffnesses $c_{1}-c_{5}$ and active SMC parameters $S_{\max }, \beta, p_{0}, \lambda_{M}, \lambda_{0}$ ) are constant across the vascular tree, but that the parameters representing the wall composition (mass fractions) and homeostatic deposition stretch of the constituents (pre-stretches) are vessel-specific (i.e., vary with vessel size and its homeostatic transmural pressure). Table 1 summarizes the parameters of the constrained mixture model and their definitions.

Table 1. Parameters defining the mechanical properties of the vessel wall model. The passive and active material parameters are constant across all representative vessels. The vessel-specific parameters vary between representative vessels with respect to their size and myocardial location.

\begin{tabular}{|c|c|c|c|}
\hline & Parameter & Description & Unit \\
\hline \multirow{5}{*}{$\begin{array}{l}\text { Passive material } \\
\text { parameters } \\
\text { (Appendix A) }\end{array}$} & $c_{1}$ & Elastin material parameter & $\mathrm{Pa} / \mathrm{kg}$ \\
\hline & $c_{2}$ & Collagen material parameter & $\mathrm{Pa} / \mathrm{kg}$ \\
\hline & $c_{3}$ & Collagen dimensionless material parameter & - \\
\hline & $c_{4}$ & SMCs material parameter & $\mathrm{Pa} / \mathrm{kg}$ \\
\hline & $c_{5}$ & SMCs dimensionless material parameter & - \\
\hline \multirow{5}{*}{$\begin{array}{c}\text { Active SMC model } \\
\text { parameters } \\
(\text { Section } 2.1)\end{array}$} & $S_{\max }$ & Total maximum stress & $\mathrm{MPa}$ \\
\hline & $\beta$ & Hill curve slope & - \\
\hline & $p_{0}$ & Hill curve offset & $\mathrm{mmHg}$ \\
\hline & $\lambda_{0}$ & Zero active tension stretch & - \\
\hline & $\lambda_{M}$ & Maximum active tension stretch & - \\
\hline \multirow{6}{*}{$\begin{array}{l}\text { Vessel-specific } \\
\text { parameters } \\
\text { (Appendix A) }\end{array}$} & $G_{\theta}^{e}, G_{Z}^{e}$ & Elastin pre-stretch & - \\
\hline & $G_{h}^{c}$ & Collagen pre-stretch & - \\
\hline & $G_{h}^{m}$ & SMCs pre-stretch & - \\
\hline & $v^{e}$ & Elastin mass fraction & - \\
\hline & $v^{c}$ & Collagen mass fraction & - \\
\hline & $v^{m}$ & SMC mass fraction & - \\
\hline
\end{tabular}

Parameter estimation: Liao and Kuo (55) measured the passive and myogenic pressure-diameter relations in four classes of swine coronary vessels with internal diameters of $180 \pm 20 \mu \mathrm{m}$ (small arteries), $95 \pm 2 \mu \mathrm{m}$ (large arterioles), $62 \pm 2 \mu \mathrm{m}$ (intermediate arterioles), and $37 \pm 2 \mu \mathrm{m}$ (small arterioles) using pressure myography. In this work, we used these relations to estimate the parameters of the constrained mixture model for the four representative vessel types in each subepicardial and subendocardial layers. To replicate the passive pressure-diameter relations, we fixed the shear-dependent and metabolic activation level and myogenic control to zero ( $A=0$ and $\left.S_{p}=0\right)$. Conversely, to mimic the myogenic data of Liao and Kuo, we assumed no contribution to vasodilation from either the shear-dependent or the metabolic mechanisms, i.e., $A=1$. In this case, the active SMC stress $S$ is given entirely by the myogenic control $S_{p}$ (Eq. 3). 
Overall, identifying the parameters of the constrained mixture model of the eight representative vessels required estimating a total of 50 parameters. Several physiological assumptions and constraints were considered to avoid unrealistic values of the model parameters. The collagen fiber angles were prescribed to follow the axial, circumferential, and diagonal directions (e.g. $0^{\circ}, 90^{\circ}$ and $45^{\circ}$, respectively). Collagen is assumed to have a relatively low pre-stretch, thus we used $0.95<G_{h}^{c}<1.05$. The pre-stretches of elastin $G_{\theta}^{e}$ and $G_{z}^{e}$ and SMCs $G_{h}^{m}$, however, were assumed to be greater than 1 (84).

The homeostatic condition of a vessel, i.e., its in-vivo diameter, thickness, and transmural pressure, is needed to identify the parameters of the constrained mixture model. In this work, we assumed an in-vivo vessel diameter $\left(D_{h}\right)$ corresponding to the experimental data (55), and obtained the vessel-specific pressure $\left(p_{h}\right)$ and thickness $(H)$ from literature $(33,62)$. Finally, the distension pressure in the Liao and Kuo experiments was always positive, and thus, the data did not describe the vessel response under compression. Therefore, in this work we assumed that the active SMC stress $S$ under compression is zero, similar to findings in skeletal muscle circulation (17). The downhill simplex method was used to estimate the parameters of the constrained mixture model (76) by minimizing the mean squared error $(E)$ between predicted and measured diameters for a given transmural pressure.

The in-vivo activation level $A$ for a given vessel should be in the range of 0 and 1 . Therefore, once the parameters associated with passive $\left(A=0\right.$ and $\left.S_{p}=0\right)$, and myogenic $(A=1)$ responses are identified, an in-vivo vessel-specific homeostatic (baseline) activation $A_{h}$ can be computed using $D_{h}$ and $p_{h}$. Consequently, the homeostatic active SMC stress $S_{h}$ can be calculated via Eq. 2: $S_{h}=A_{h} \times S_{p}\left(p_{h}\right)$.

Once the parameters of the constrained mixture model (Table 1) for the representative vessels (4 in subendocardial and 4 in subepicardial layer) are identified, they are assigned to each individual vessel of the coronary trees according to its size.

Sensitivity analysis: To understand the sensitivity of the estimated parameters of the constrained mixture model, a sensitivity analysis was performed following the approach of Pradhan and colleagues (69). A sensitivity index $X$ was defined as the percentage change in model error $E$ (Appendix B) associated with introducing a $10 \%$ change in the parameter values one at a time

$$
X=\frac{100}{E(\theta)}|E(\theta+0.1 \theta)-E(\theta)|
$$

where $\theta$ is the estimated parameter value, and $E(\theta)$ is its associated error, defined as the difference between predicted and measured diameters.

\subsection{Defining morphometry, structure, and hemodynamics of an entire coronary tree}

The second stage is to generate two coronary trees in the subendocardial and subepicardial layers and to estimate their homeostatic baseline state, using the homeostatic optimization framework developed by (29). The approach of Filonova et al. is a framework to estimate the homeostatic morphometric (e.g, diameters), structural (e.g, thickness), and hemodynamics (e.g., wall shear stress) properties of a vascular tree by minimizing the global energy dissipation under the constraint of mechanical equilibrium. This energy minimization is performed via adjustments of 2 parameters in each vessel segment of the tree: 1) the homeostatic diameter of the vessel and, 2) the amount of the materials of the vessel wall that constitute the thickness (elastin, collagen, SMCs) (Appendix C). 
Tree initialization: First, two symmetric trees (subendocardial and subepicardial) were initialized using morphometry data. The parent-to-daughter relationship is used to describe the branching pattern of the vascular trees

$$
D_{p}^{\xi}=D_{d 1}^{\xi}+D_{d 2}^{\xi}
$$

where $D_{p}, D_{d 1}$, and $D_{d 2}$ are the diameters of the parent and two daughter vessels, respectively, and $\xi$ is called the diameter exponent. Vessels feeding the intramyocardial arteries of the left ventricular free wall are $438 \pm 56 \mu \mathrm{m}$ in diameter (42). With an initial radial exponent of $\xi_{0}=2.55$ (46), the diameter of the firstgeneration vessel equal to $438 \mu \mathrm{m}$ and the diameter of the last generation vessels $\sim 20 \mu \mathrm{m}$, the number of generations in the trees is 11 . Vessel lengths were determined as a function of vessel diameters using the morphometric data in Kassab et al. (48). The subendocardial and subepicardial trees were assumed to be located at $5 / 6$ and $1 / 6$ of the myocardial depth, respectively.

Tree optimization: We considered hemodynamic steady state conditions to define baseline homeostatic characteristics of the trees, with distinct mean intramyocardial pressures for each tree. Using a thin-walled assumption, the mechanical equilibrium in each artery can be expressed via Laplace's law:

$$
p_{t m} D / 2=T_{\theta \theta},
$$

where $p_{t m}$ is the transmural pressure of the myocardial blood vessels, $T_{\theta \theta}$ is the arterial membrane stress that incorporates the mechanical contributions of elastin, collagen, and SMCs (Appendix A), and $D$ is the inner diameter of the segment. The transmural pressure can be written as

$$
p_{t m}=p-p_{\text {im }}
$$

where $p$ is the luminal pressure and $p_{i m}$ is the intramyocardial pressure. Algranati and colleagues (3) postulated that $p_{i m}$ is a function of the cavity-induced extracellular pressure $\left(p_{C E P}\right)$ and muscle shorteninginduced pressure $\left(p_{S I P}\right)$ :

$$
p_{\text {im }}=p_{C E P}+p_{S I P}
$$

Dependence on $p_{C E P}$ implies that the compressive forces are larger in the inner layers (endocardial) of the left ventricle (LV) than those in the outer (epicardial) layers (7). Furthermore, it has been established that this variation in myocardial pressure affects the flow distribution and structure of the arteries in different layers (50). We assumed that for the free wall of the LV, $p_{C E P}$ varies linearly from endocardium (LV pressure, $p_{L V}$ ) to epicardium, where the pericardial pressure is assumed to be negligible. Therefore, for the subendocardial and subepicardial trees, the average $p_{C E P}$ values were set to $5 / 6$ and $1 / 6$ of $p_{L V}$, respectively, consistent with their relative myocardial depth. The muscle shortening- induced pressure $p_{S I P}$ was chosen so that subendocardium $p_{i m}$ is $\sim 20 \%$ larger than the $p_{L V}(65)$.

The boundary conditions (i.e., hemodynamic constraints) of the homeostatic optimization in both subepicardial and subendocardial trees are the inlet and outlet pressures. In general, imposing only pressure boundary conditions is not sufficient for the homeostatic optimization as the solution will not converge (29). Therefore, a myocardial layer-dependent total flow in the arterial tree was considered as a soft constraint for the global optimization, by adding a penalty term to the cost function (Appendix C). A downhill simplex method was used for the minimization of the global cost function. The direct result of the minimization is the diameter of the vessels in coronary trees. Once the vessel diameter is known, the vessel wall thickness can be computed as a post-processing using the mechanical equilibrium equation (Appendix A). 
Model parameters: In this work, the bulk of model parameters corresponds to literature swine data, with the exception of flow at terminal arterioles, which corresponds to canine data, given lack of measurements for swine (67). Next, we detail the data used to set our hemodynamics and how the constrained mixture model parameters are assigned to the coronary tree vessels.

The hemodynamics parameters used for generating the coronary trees are listed in Table 2. Baseline coronary pressure was taken to be the mean aortic pressure $100 \mathrm{mmHg}$ since the pressure drop in epicardial arteries, in absence of occlusive disease, is negligible. The outlet pressure at the terminal vessels (small arterioles) was set to $55 \mathrm{mmHg}(27,72)$. Based on blood flow velocity measurements in $(8,78,82)$, a baseline flow rate of $3 \times 10^{-3} \mathrm{~mm}^{3} / \mathrm{s}$ was used for the subepicardial terminal arterioles. To determine the flow for the subendocardial tree, the total subendocardial to subepicardial flow ratio (ENDO/EPI) was assumed to be $\sim 1.25$ (22). Intramyocardial pressures $p_{i m}$ of 47 and $13 \mathrm{mmHg}$ were imposed on subendocardial and subepicardial vessels, respectively. A diameter dependent blood flow viscosity model was used based on (73) (Appendix D).

Following (18), the constrained mixture model parameters were assigned to the coronary vessels according to their sizes (Arteries: $\mathrm{D}>190 \mu \mathrm{m}$, Large Arterioles: $190 \geq \mathrm{D}>100 \mu \mathrm{m}$, Intermediate Arterioles: $100 \geq \mathrm{D}>50$, Small Arterioles: 50 $\geq$ D) for each tree. This classification is limited by the experimental data and is relatively crude since the pressures and wall thicknesses within one class of vessels may vary significantly. For instance, our initial analysis showed that when all the parameters are constant across a class type, the homeostatic optimization framework does not produce the correct thickness-to-diameter ratio trends when compared to experimental data. Therefore, we adjusted the baseline activation level of SMCs $\left(0<A_{h}<\right.$ 1 ), which is the in-vivo value of $A$ in Eq. 2, for each vessel to match the thickness-to-diameter ratio with data in (33), as demonstrated in the results section.

Table 2. Tree initialization and hemodynamic parameters for generation of coronary trees.

\begin{tabular}{|c|c|c|c|c|}
\hline Parameter & Description & Value & Unit & Ref \\
\hline$\xi_{0}$ & Initial diameter exponent & 2.55 & - & (46) \\
\hline$D_{0}$ & $\begin{array}{l}\text { Initial first-generation } \\
\text { diameter }\end{array}$ & 438 & $\mu \mathrm{m}$ & $(42)$ \\
\hline$p_{\text {in }}$ & Inlet coronary pressure & 100 & $\mathrm{mmHg}$ & \\
\hline$p_{\text {out }}$ & Terminal pressure & 55 & $\mathrm{mmHg}$ & $(27,72)$ \\
\hline$q_{\text {subepi }}$ & $\begin{array}{c}\text { Subepicardial (baseline) } \\
\text { terminal flow }\end{array}$ & $3 \times 10^{-3}$ & $\mathrm{~mm}^{3} / \mathrm{s}$ & $(8,78,82)$ \\
\hline ENDO/EPI & $\begin{array}{c}\text { Subendocardial to } \\
\text { subepicardial flow ratio }\end{array}$ & 1.25 & - & Duncker and Bache 2008) \\
\hline $\begin{array}{l}p_{\text {im }} \text { (subepi., } \\
\text { subendo.) }\end{array}$ & Intramyocardial pressure & 13,47 & $\mathrm{mmHg}$ & $(65)$ \\
\hline
\end{tabular}

\subsection{Coronary autoregulation model}

The third stage of the proposed model is to develop a structurally-motivated coronary autoregulation model based on constrained mixture theory. In coronary autoregulation, vessel reactivity to changes in pressure is mediated by the active SMC stress $S$ (Eq. 2). Although autoregulation is a dynamic process involving the activation of each of the metabolic, shear-dependent and myogenic mechanisms with their respective time 
responses, in this work we are concerned with the steady state reached within minutes following a perturbation from a previous state $(5,19,66)$.

A key component of the proposed autoregulation model is the activation function $A$ (Eq. 2), which determines the activation level from a fully dilated $(A=0)$ to a maximum myogenic response $(A=1)$. The myogenic control $S_{p}$, is a constrictive response to increases in the local wall stress resulting from changes in transmural pressure $p_{t m}$. The myogenic control is formulated by the sigmoidal function in Eq. 3 .

In shear-dependent control, an increase in wall shear stress $\tau$ induces relaxation of the SMCs mediated by upregulation of endothelial NO synthesis. This vasodilatory stimulus $s_{\tau}$ is modeled as

$$
s_{\tau}=-a_{\tau}\left(\frac{\tau-\tau_{h}}{\tau_{h}}\right)
$$

where $\tau_{h}$ is the baseline (homeostatic) value of wall shear stress, and $a_{\tau}$ is the shear-dependent scaling coefficient. The negative sign indicates a decrease in vessel activation for an increase in $\tau$.

Moreover, experimental studies show that the oxygen extraction capacity of the myocardium is almost fully exhausted in both rest and exercise (24). Therefore, we assume that changes in $\mathrm{MVO}_{2}$ are matched by proportional changes in flow to supply the oxygen demand (6). Based on this assumption, we take the terminal flow as a measure of metabolic signal, regardless of the underlying physiological mechanisms involved (66), and write the metabolic stimuli as

$$
s_{m}=-a_{m}\left(\frac{\hat{q}\left(M V O_{2}\right)-q_{t e r m}}{\hat{q}\left(M V O_{2}\right)}\right),
$$

where $\hat{q}\left(\mathrm{MVO}_{2}\right)$ is the target flow determined by baseline metabolic demand, $q_{\text {term }}$ is the actual flow rate at the terminal arterioles, and $a_{m}$ is the metabolic scaling coefficient. The negative sign indicates a decrease in metabolic stimuli for a decrease in terminal flow. It must be noted that in this work, we focus on the coronary autoregulation for a fixed baseline myocardial activity, i.e., $\hat{q}\left(\mathrm{MVO}_{2}\right)$ remains constant.

To compute the total stimuli induced by the shear-dependent and metabolic control mechanisms, two factors must be considered. First, the magnitude of each control mechanism depends of the vessel size. Sheardependent vasodilation is mostly blunted in vessels smaller than $100 \mu \mathrm{m}$ (78). Meanwhile, the metabolic response from different signaling pathways (e.g., oxygen demand/supply) is initiated in capillaries, and it is conducted and integrated upstream to precapillary arterioles (5). The integrated response, however, decays exponentially so that it mostly affects the intermediate and small arterioles with a peak at vessels $\sim 50 \mu \mathrm{m}$. Second, the equations 9 and 10 only describe the stimuli when a deviation from homeostatic conditions occurs. However, SMCs maintain a basal tone under resting conditions (59). Therefore, the total SMC stimuli for each vessel can be expressed as a basal stimulus plus the superposition of the two stimuli described above, viz.:

$$
s_{\text {total }}=\phi_{\tau}(D) s_{\tau}+\phi_{m}(D) s_{m}+s_{h}
$$

where $s_{h}$ is a SMC basal (homeostatic) stimuli. Based on the experimental observations $(13,45,53,54)$, the diameter-dependent weights $\phi_{\tau}$ and $\phi_{m}$, representing the magnitude of each mechanism in the total stimulation, are prescribed (Fig. 2)

$$
\phi_{\tau}(D)=\frac{1}{1+\mathrm{e}^{b_{1}\left(D-b_{2}\right)}}, \text { and } \phi_{m}(D)=\frac{1}{\mathrm{e}^{\left(b_{3}\left(D-b_{4}\right)\right)^{2}}},
$$


where $b_{1}$ and $b_{3}$ are 0.02 and $0.0131 / \mu \mathrm{m}$, and $b_{2}$ and $b_{4}$ are 250 and $50 \mu \mathrm{m}$, respectively. Following (15), a sigmoidal function is used to relate the total stimuli $s_{\text {total }}$ to the activation function $A$ :

$$
A=\frac{1}{1+e^{-s_{\text {total }}}}
$$

Therefore, the basal stimuli $s_{h}$ for each vessel in the coronary tree can be calculated using the homeostatic activation level $A_{h}$

$$
s_{h}=-\log \left(\frac{1-A_{h}}{A_{h}}\right)
$$

Combining equations (2)-(3),(9)-(13), we obtain expression for the total active SMC stress $S$ as a function of $p_{t m}, \tau$, and $\mathrm{MVO}_{2}$.

$$
S=\frac{1}{1+e^{-\left\{-\phi_{\tau}(D) a_{\tau}\left(\frac{\tau-\tau_{h}}{\tau_{h}}\right)-\phi_{m}(D) a_{m}\left(\frac{\hat{q}\left(M V O_{2}\right)-q_{t e r m}}{\hat{q}\left(M V O_{2}\right)}\right)+s_{h}\right\}}} S_{\max } \frac{p_{t m}^{\beta}}{p_{t m}^{\beta}+p_{0}^{\beta}} .
$$

The scaling coefficients $a_{\tau}$ and $a_{m}$ for the autoregulation model are estimated using the collection of swine coronary autoregulation data in (21). The procedure and the results of this calibration are described in Section 3.3. Table 3 summarizes the model parameters for the autoregulation model and how the values are estimated/prescribed.

Table 3. Autoregulation model parameters

\begin{tabular}{ccccc}
\hline Parameter & Description & Unit & \\
\cline { 1 - 3 }$a_{\tau}$ & Shear-dependent control scaling coefficient & - & Fit to experimental data \\
Figure 9
\end{tabular}




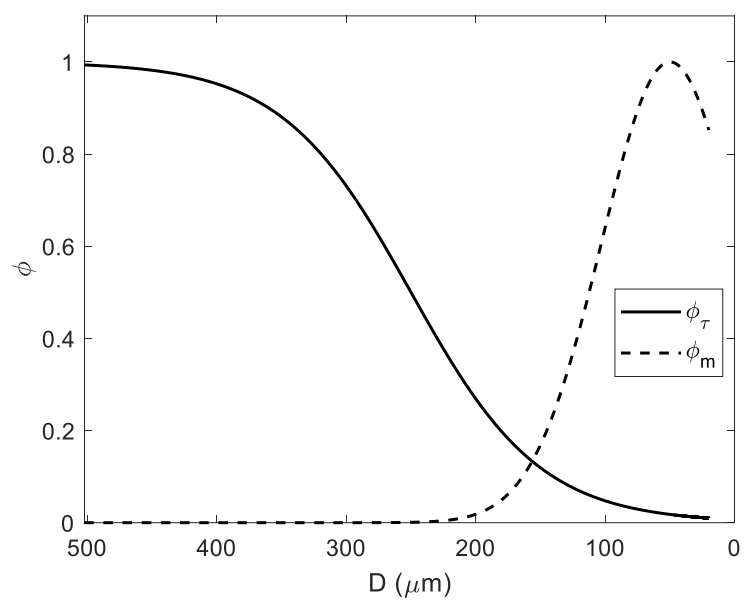

Figure 2. Magnitude of the shear-dependent and metabolic mechanisms, based on $(13,45,53,54)$. The shear-dependent mechanism is weak in arterioles smaller than $100 \mu \mathrm{m}$. A metabolic signal is initiated at the end of terminal vessels (capillaries/venules) and is conducted upstream to coronary arterioles, where signals from different terminal vessels are integrated. The metabolic signal, however, decreases exponentially for larger vessels. The magnitude of the metabolic mechanism was assumed to be highest at the arterioles (peak at $50 \mu \mathrm{m}$ ).

The coronary pressure will therefore critically determine the relative contribution of the different control mechanisms down the coronary tree. Pressure and flow rates are calculated in each individual vessel of the entire coronary tree. A change in input coronary pressure elicits a new hemodynamic state and corresponding stimuli from each control mechanism. New SMC activation levels and active SMC stress are computed and used in the constrained mixture model to determine the changes in vessel size, which in turn result in an updated global hemodynamic state. This process is repeated until the tree hemodynamics are converged, which defines the new regulated state. To ensure the uniqueness of the converged steady state, the initial conditions for the global steady state hemodynamics were randomly varied. This workflow is depicted in Fig. 3.

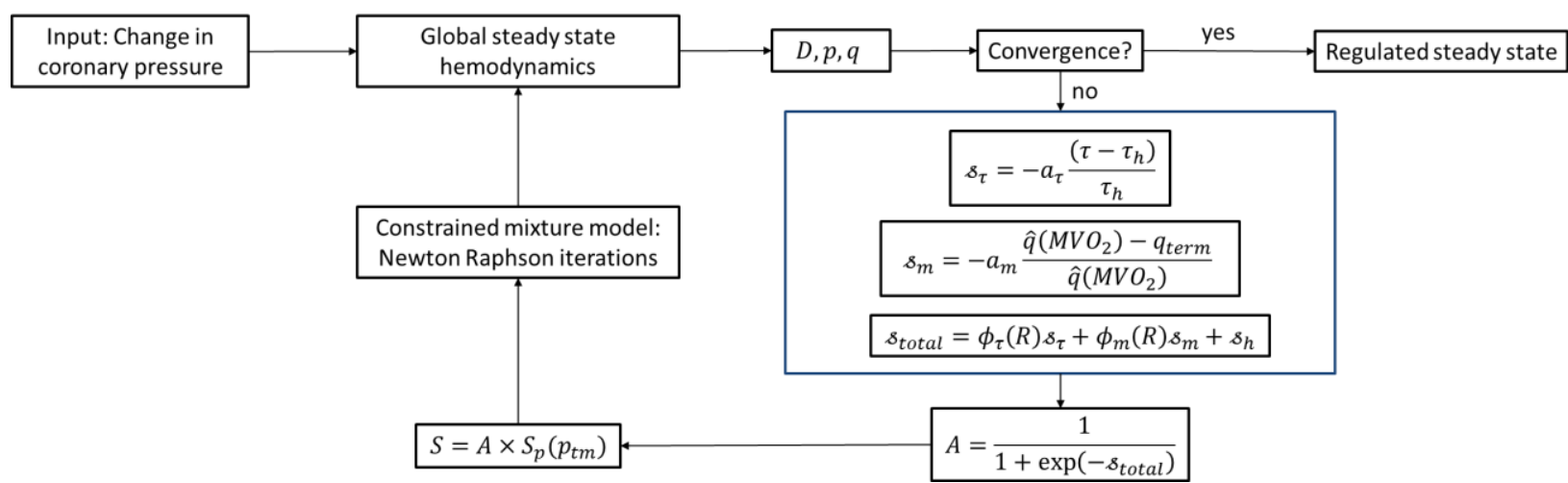

Figure 3. The workflow of the autoregulation model. The model input is the coronary pressure $p_{i n}$. Convergence in hemodynamics (pressure and flow in the entire tree) defines a new regulated state. 


\section{Results}

In Sections 3.1 and 3.2, we present the results of model calibration for the constrained mixture model parameters and for the morphometry, structure, and hemodynamics of the different coronary trees, respectively. In Section 3.3, we illustrate the autoregulation model by calculating responses every $5 \mathrm{mmHg}$ of input coronary pressure for the $20-160 \mathrm{mmHg}$ pressure range. All the stages of the model were implemented in MATLAB (Mathwork, Natick, MA).

\subsection{Constrained mixture model parameters}

Table 4 and Fig. 4 show the constrained mixture model parameter values estimated using the experiment data from Liao and Kuo (55) depicted in Fig. 5. Relatively higher mass fractions of SMCs were estimated in the large and intermediate arteriolar levels compared to small arteries and small arterioles for both coronary trees (Fig. 4). In addition, the pre-stretch of collagen fibers in the small arterioles of subepicardium and all arterioles of subendocardium were estimated to be less than 1, which means that these fibers are slightly compressed in the homeostatic condition of the vessel (Table 4). The SMC passive material parameters show the lowest sensitivity (see sensitivity indices in Table 4). In Fig. 5, the model results are compared with experimental data for passive $\left(A=0\right.$ and $\left.S_{p}=0\right)$ and myogenic $\left(A=1\right.$ and $S_{p}$ from Eq. 3) responses.
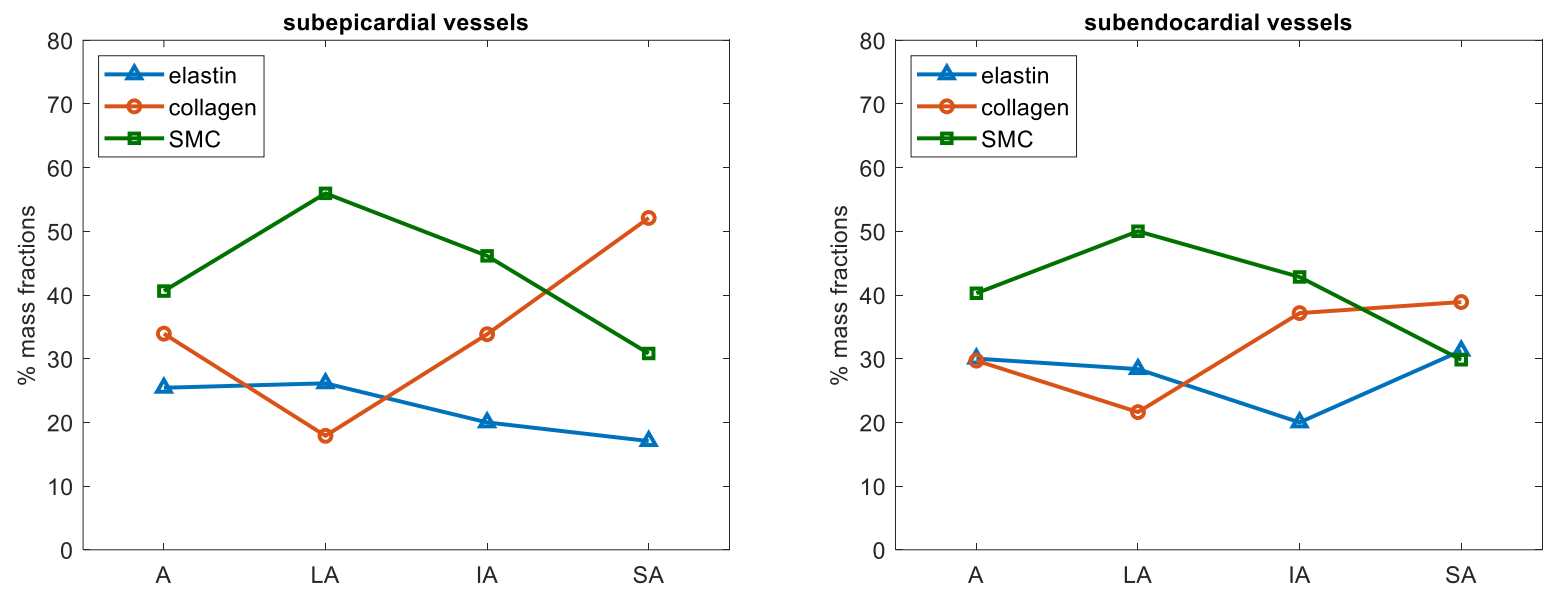

Figure 4. Estimated mass fractions $\left(v^{e}, v^{c}\right.$, and $\left.v^{m}\right)$ for the different vessel types in our model. The mass fraction of SMCs seems to be the highest in large arterioles in both subendocardial and subepicardial layers. (A: Small arteries, LA: Large, IA: Intermediate, SA: Small arterioles.)

Table 4. Constrained mixture model parameters, and their estimated value and sensitivity index. Experimental data shown in Fig. 5 were used for estimation of the parameters.

\begin{tabular}{cccc}
\hline & Parameter & Value & Sensitivity Index $X(\%)$ \\
\hline & $c_{1}(\mathrm{~Pa} / \mathrm{kg})$ & 113.60 & 0.8 \\
\cline { 2 - 4 } & $c_{2}(\mathrm{~Pa} / \mathrm{kg})$ & 333.69 & 3.2 \\
\cline { 2 - 4 } $\begin{array}{c}\text { Passive material } \\
\text { parameters }\end{array}$ & $c_{3}$ & 4.64 & 8.0 \\
\cline { 2 - 4 } & $c_{4}(\mathrm{~Pa} / \mathrm{kg})$ & 78.21 & 0.2 \\
\cline { 2 - 4 } & $c_{5}$ & 0.42 & 0.1 \\
\hline & $S_{\max }(\mathrm{MPa})$ & 2.45 & 10.9 \\
\hline
\end{tabular}


bioRxiv preprint doi: https://doi.org/10.1101/2020.09.21.304030; this version posted September 22, 2020. The copyright holder for this preprint (which was not certified by peer review) is the author/funder, who has granted bioRxiv a license to display the preprint in perpetuity. It is made available under aCC-BY-ND 4.0 International license.

\begin{tabular}{|c|c|c|c|}
\hline \multirow{5}{*}{$\begin{array}{c}\text { Active material } \\
\text { parameters }\end{array}$} & \\
\hline & $\beta$ & 1.90 & 10.5 \\
\hline & $p_{0}(\mathrm{mmHg})$ & 70.34 & 33.6 \\
\hline & $\lambda_{0}$ & 0.5 & 25.6 \\
\hline & $\lambda_{M}$ & 1.7 & 39.3 \\
\hline \multirow{6}{*}{$\begin{array}{l}\text { Subepicardial } \\
(\mathrm{A}, \mathrm{LA}, \mathrm{IA}, \mathrm{SA})^{*}\end{array}$} & $G_{\theta}^{e}, G_{Z}^{e}$ & $(1.22,1.12,1.06,1.06)$ & $(6.4,0.5,2.8,0.8)$ \\
\hline & $G_{h}^{c}$ & $(1.05,1.05,1.00,0.98)$ & $(14.3,15.6,11.2,7.7)$ \\
\hline & $G_{h}^{m}$ & $(1.05,1.05,1.05,1.05)$ & $(2.3,1.3,2.1,1.3)$ \\
\hline & $v^{e}$ & \multirow{3}{*}{ Shown in Figure 4} & $(6.2,0.8,0.5,0.1)$ \\
\hline & $v^{c}$ & & $(10.6,2.0,1.6,2.2)$ \\
\hline & $v^{m * *}$ & & - \\
\hline \multirow{6}{*}{$\begin{array}{l}\text { Subendocardial } \\
\text { (A,LA,IA,SA) }\end{array}$} & $G_{\theta}^{e}, G_{z}^{e}$ & $(1.15,1.10,1.02,1.02)$ & $(6.0,1.6,0.9,4.6)$ \\
\hline & $G_{h}^{c}$ & $(1.02,0.99,0.95,0.97)$ & $(15.2,14.4,13.0,9.6)$ \\
\hline & $G_{h}^{m}$ & $(1.05,1.05,1.05,1.02)$ & $(1.2,2.0,2.3,1.7)$ \\
\hline & $v^{e}$ & \multirow{3}{*}{ Shown in Figure 4} & $(9.1,1.9,1.4,0.5)$ \\
\hline & $v^{c}$ & & $(10.6,2.0,3.8,0.9)$ \\
\hline & $v^{m}$ & & - \\
\hline
\end{tabular}

*A: Arteries, LA: Large, IA: Intermediate, SA: Small arterioles.

$* * v^{m}=1-\left(v^{c}+v^{e}\right)$ 
bioRxiv preprint doi: https://doi.org/10.1101/2020.09.21.304030; this version posted September 22, 2020. The copyright holder for this preprint (which was not certified by peer review) is the author/funder, who has granted bioRxiv a license to display the preprint in perpetuity. It is made available under aCC-BY-ND 4.0 International license.

Subepicardial
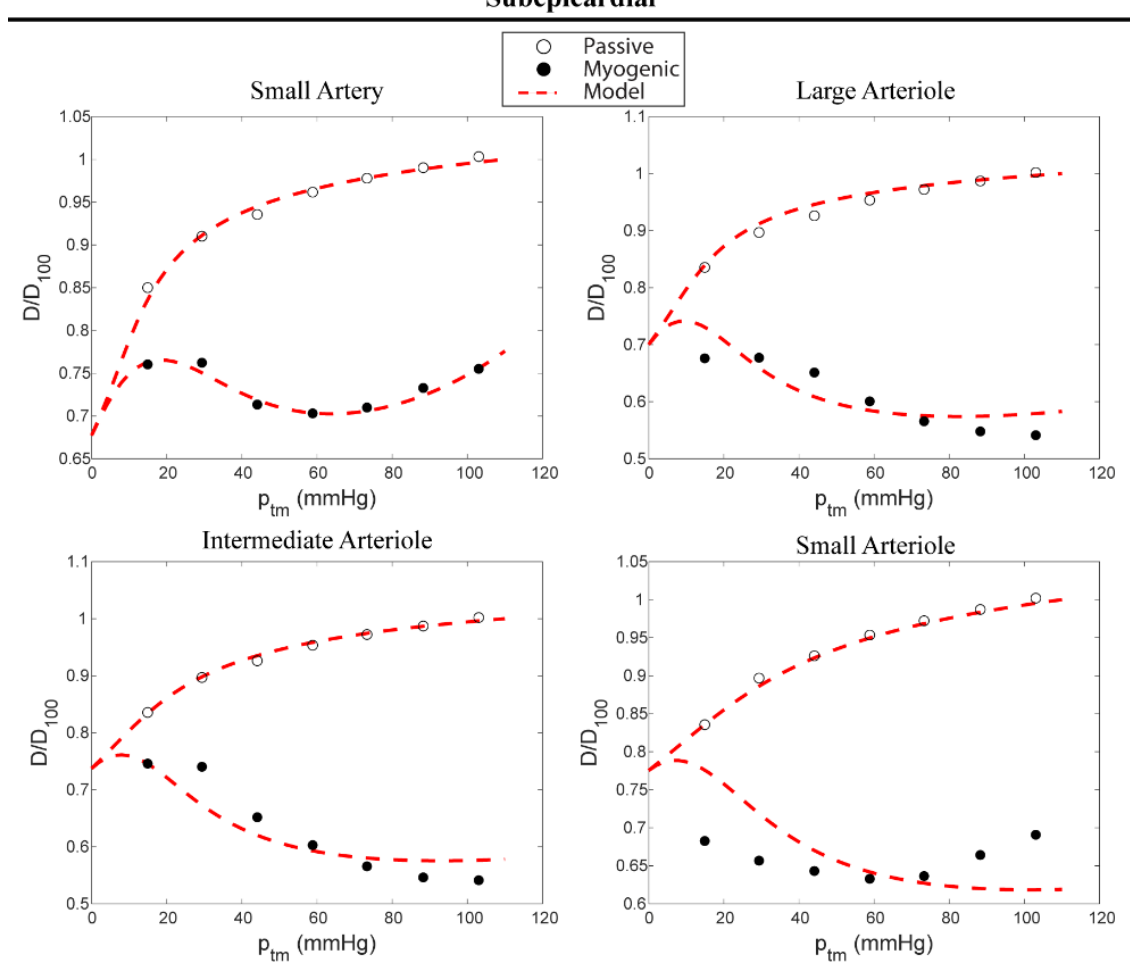

Subendocardial
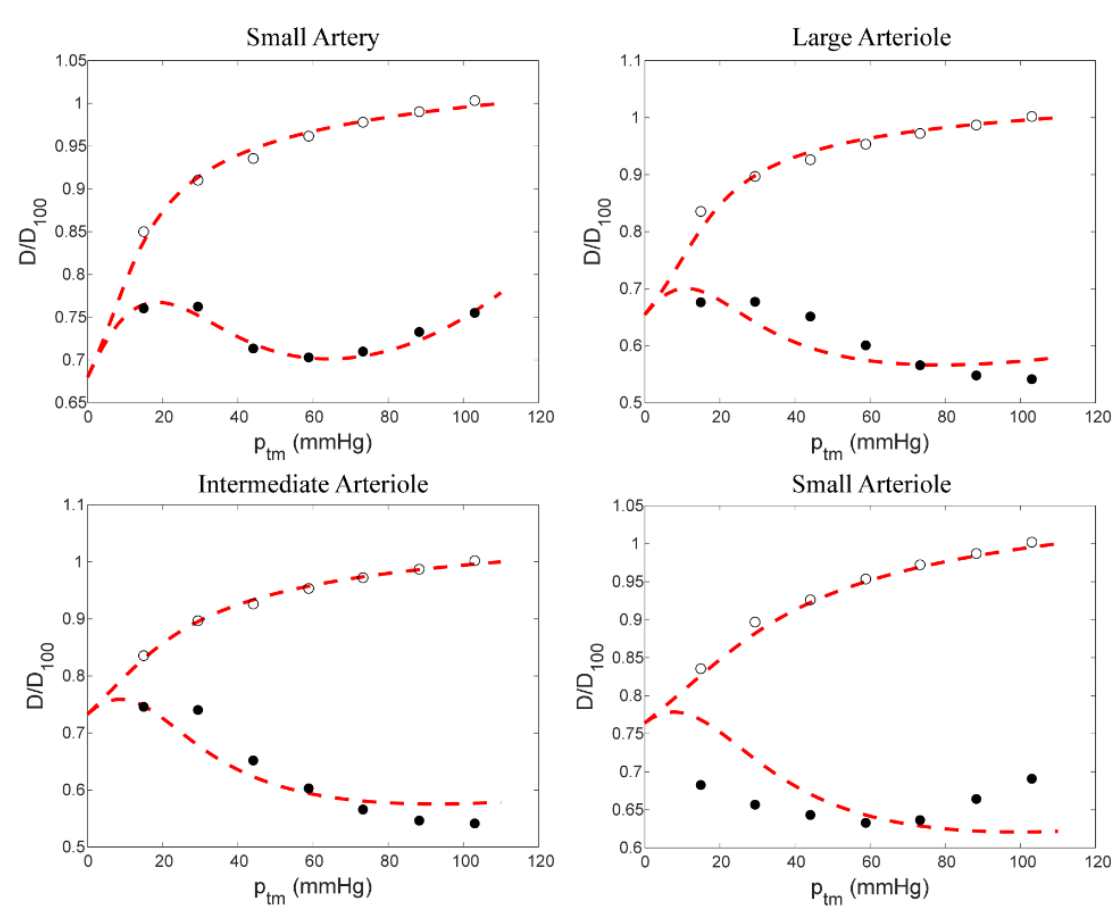

Figure 5. Data on passive and myogenic responses (55), and model fits for the representative vessels in the subepicardial (top) and subendocardial (bottom) trees. The parameters listed in Table 1 are estimated by minimizing the mean squared error between the predicted and measured diameters at the given values of transmural pressure estimate. $D_{100}:$ Passive diameter at $103 \mathrm{mmHg}\left(100 \mathrm{cmH}_{2} \mathrm{O}\right.$ in the experiments.) 


\subsection{Morphometry, structure, and hemodynamics of an entire coronary tree}

Morphometric results of the homeostatic optimization are summarized in Fig. 6. The homeostatic optimization estimates vessel diameters $D_{h}$, determines bifurcation rules, identifies vessel types based on their sizes, and assigns the appropriate mechanical parameters (29). Subendocardial vessels appear to be larger than their subepicardial counterparts. This difference, consistent with experimental and modeling results from $(3,35)$, facilitates a higher blood flow in the subendocardial than in the subepicardial layer. Furthermore, our results indicate an increasing diameter exponent $\xi(2.5-2.7)$ with generation number for both trees.
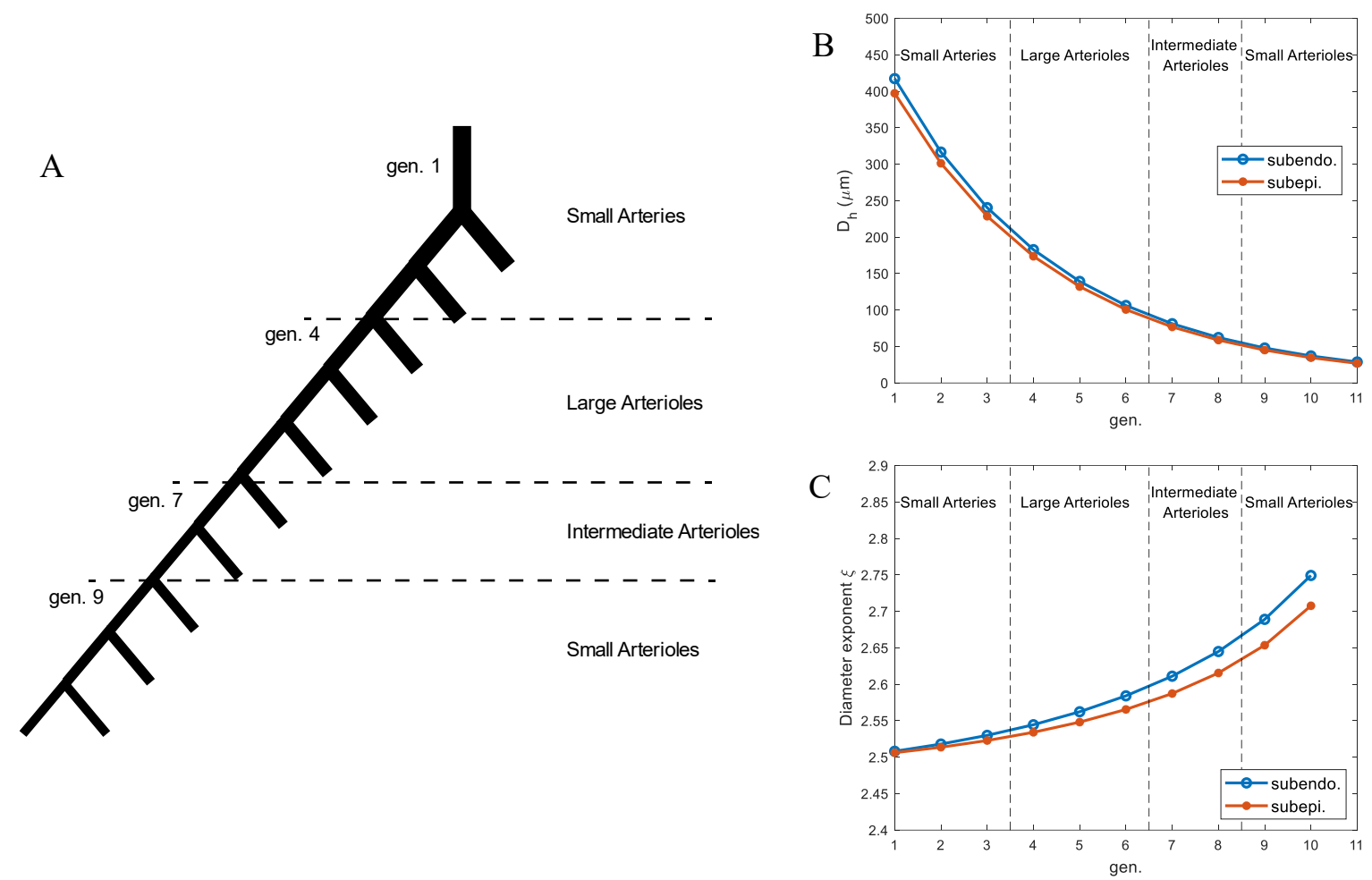

Figure 6. A: Schematic of a symmetric coronary tree, identifying the vessel types in each generation (number of generations and vessel classifications are the same in both subendocardial and subepicardial trees). B: Homeostatic vessel diameter $D_{h}$ across generations for the subendocardial and subepicardial trees. C: Diameter exponent in daughter-toparent diameter relation $\left(D_{p}^{\xi}=D_{d 1}^{\xi}+D_{d 2}^{\xi}\right)$ across generations for the subendocardial and subepicardial trees.

Figure 7 shows the structural and hemodynamics results of the homeostatic optimization for both subendocardial and subepicardial trees, and comparisons with available experimental data. Figure 7-A shows our results for thickness to diameter ratio for all generations. Figure 7-B shows pressure across the trees. It is apparent that most of the coronary vascular resistance is in arterioles $<100 \mu \mathrm{m}$. This range seems to be similar across different organs and species. Consistently, VanBavel and Spaan (86) showed that pressure in the coronary microvasculature drops from 90 to $30 \mathrm{mmHg}$ in $\sim 10-\mu \mathrm{m}$ diameter vessels. Wall shear stress increases almost 3-fold in arterioles, consistent with experimental studies (Fig. 7-C). Figure 7D shows the homeostatic circumferential stress computed from Laplace's law $\left(\sigma_{h}=p_{t m} D /(2 H)\right)$, where $H$ is the vessel wall thickness. Since the intramyocardial pressure is smaller towards the epicardium (resulting in higher transmural pressure), the subepicardial homeostatic circumferential stress is larger. Guo and Kassab (33) analyzed the circumferential stress in the swine coronary arterial tree and reported the circumferential stress in the range of 9.4-159 for arteries of 10-3,000 $\mu \mathrm{m}$. Although our findings show the 
same trend, the Guo and Kassab experiments correspond to an entire coronary tree (not divided between subendocardial and subepicardial regions) and were conducted in an arrested heart with no intramyocardial pressure, therefore rendering larger transmural pressures and circumferential stresses.

A

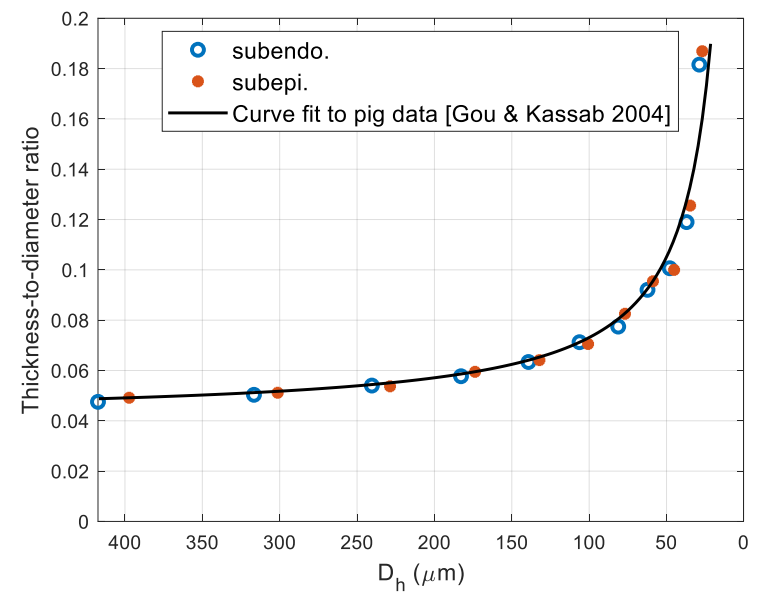

$\mathrm{C}$

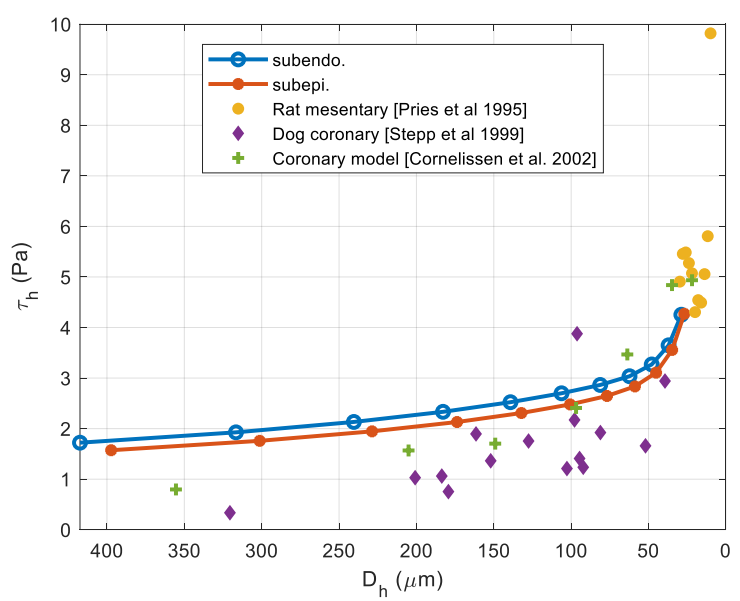

B

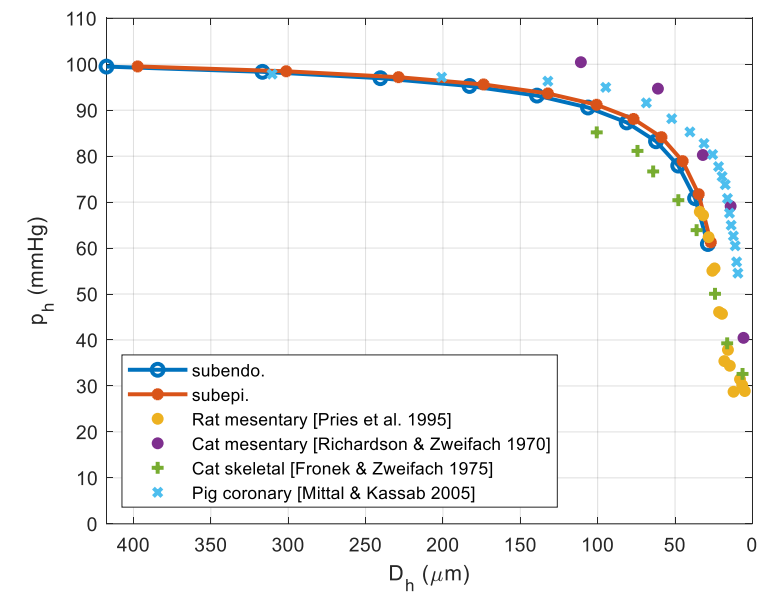

$\mathrm{D}$

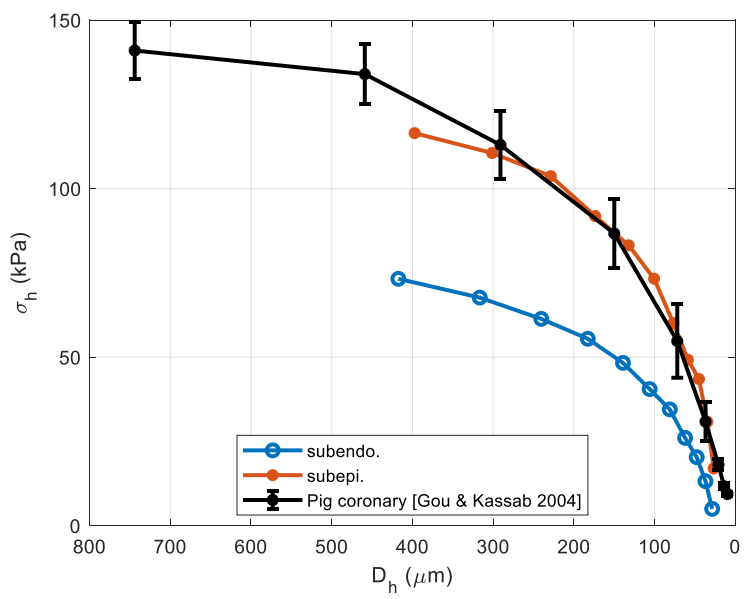

Figure 7. Homeostatic optimization results plotted against diameters: A: wall thickness-to-diameter ratio; The thickness to diameter ratio increases as the vessels become smaller. B: mid-artery pressure; Most of the coronary vascular resistance resides in arterioles $<100 \mu \mathrm{m}$. C: homeostatic value of wall shear stress $(\tau)$; A 3-fold in wall shear stress is predicted in the arterioles. D: homeostatic value of circumferential stress $\sigma_{h}$; larger transmural pressures in the subepicardial layer compared to subendocardial layer result in larger homeostatic stresses in the blood vessels.

The homeostatic activation level $\left(A_{h}\right)$ and normalized active stress of the SMCs $\left(S_{h} / S_{\max }\right)$ across both coronary trees are shown in Fig. 8. The activation level decreases from the arteries to large arterioles (Fig. 8-A). A key outcome of the homeostatic optimization is that there is a trade-off between the SMC mass fraction and the activation level in the small arteries and large arterioles, where the pressure remains relatively constant. For instance, a higher SMCs content in the large arterioles (Fig. 4) results in a decrease in activation level compared to small arteries. However, for the intermediate and small arterioles, the transmural pressure decreases drastically across the vessel generations. The homeostatic activation shows different patterns for the subendocardial and subepicardial tress. Given that the SMC mass fractions are similar for both trees, we argue that the different activation level could be due to the substantially different transmural pressures due to the much larger intramyocardial pressures in the subendocardial region. 
Furthermore, a high level of baseline activation in the small subendocardial arterioles $(\sim 0.8)$ is multiplied by a small (myogenic) pressure-dependent active stress $S_{p}$ due to a small transmural pressure, and results in a small active SMC stress $\left(S_{h} / S_{\max }<0.2\right)$ (Fig. 8-B). Overall, the homeostatic active SMC stress $S_{h}$ decreases due to large decreases in transmural pressure (Fig. 8-B).

A

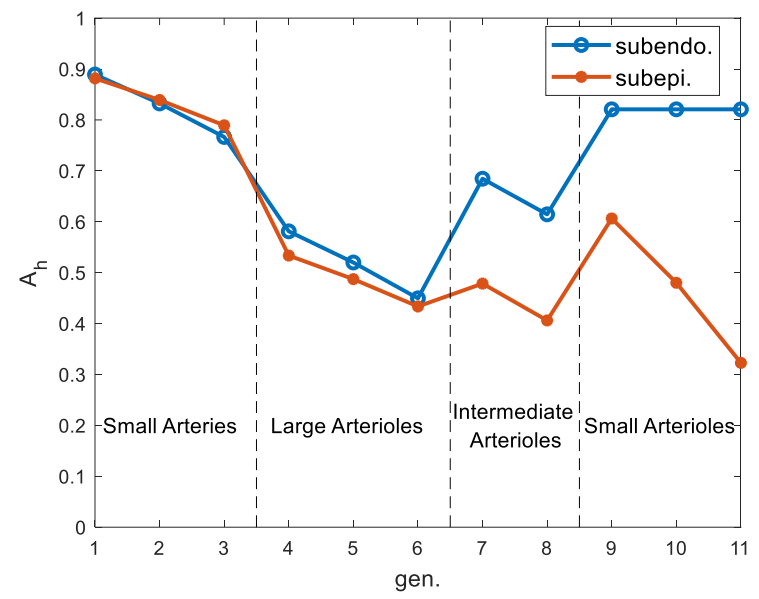

B

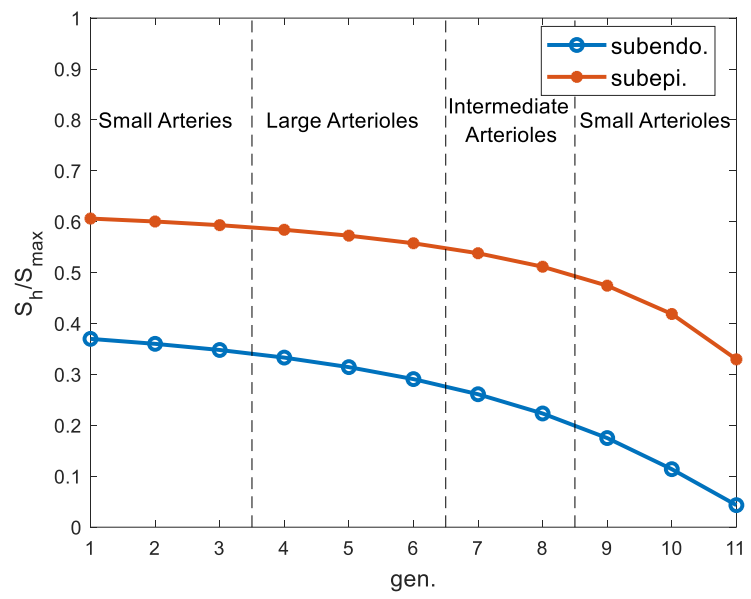

Figure 8. The homeostatic activation level $\left(A_{h}\right)$ and normalized active SMC stress $\left(S_{h} / S_{\text {max }}\right)$ across both coronary trees.

\subsection{Autoregulation model}

Before presenting the results of the coronary autoregulation model, we must note that the outlet pressure prescribed in the homeostatic optimization was a constant pressure $(55 \mathrm{mmHg})$ at the small arteriolar level. However, to extend the applicability of our coronary autoregulation model to situations of lower input coronary pressures $\left(p_{i n}<55 \mathrm{mmHg}\right)$, such as those distal to a severe occlusion in the large coronary arteries, we replaced the constant outflow pressure boundary condition with a constant resistance $R_{\text {coronary }}$ (with no vasoreactivity) boundary condition plus a $20 \mathrm{mmHg}$ terminal coronary venular pressure (72). The values of these resistances were computed using the terminal arterioles pressure minus the venular pressure, divided by the terminal homeostatic flow in the coronary trees (viz., $R_{\text {coronary }}=\Delta P / q_{\text {term }}$ ).

\subsubsection{Calibration of stimuli, activation, and active stress}

The scaling coefficients for the shear-dependent $a_{\tau}$ and metabolic $a_{m}$ controls, were determined using swine pressure-flow autoregulation data (21) and a simplex method. As a constraint for the estimation of these parameters, we assumed that the SMC activation in small arteriolar level is less than 5\% when coronary pressure falls under $40 \mathrm{mmHg}$ (31). This physiologically motivated constraint helps to deal with high degree of underdetermination of the system. The estimated scaling coefficients are summarized in Table 5.

Table 5. Estimated scaling coefficients and their sensitivity index for the autoregulatory response. Experimental data shown in Fig. 9 were used for estimation of the parameters.

\begin{tabular}{lcc}
\hline & $a_{\tau}(X \%)$ & $a_{m}(X \%)$ \\
\hline Subepicardial & $1.03(0.3)$ & $10.34(1.0)$ \\
Subendocardial & $0.98(0.3)$ & $6.92(2.6)$ \\
\hline
\end{tabular}


Figure 9 shows the results of the calibration of our coronary autoregulation model, together with the predicted pressure-flow relations in fully dilated coronary trees. The ratio of the total flow in the coronary arteries $(q)$ over the baseline (homeostatic) flow $\left(q_{h}\right)$ is given as a function of the input coronary pressure $p_{i n}$. In the range of 70-140 $\mathrm{mmHg}$ inlet coronary pressure, over which coronary autoregulation is apparent, our model fits the experimental observations well (maximum error: $22 \%$ at $140 \mathrm{mmHg}$ ). Conversely, our model predictions are less accurate in the $20-60 \mathrm{mmHg}$ inlet coronary pressure range (maximum errors of $43 \%$ at $\sim 50 \mathrm{mmHg}$ inlet pressure). In low coronary pressures $p_{\text {in }}<60 \mathrm{mmHg}$, the predicted autoregulation curve is close to the fully dilated pressure-flow relation, indicating a full dilation of the trees.

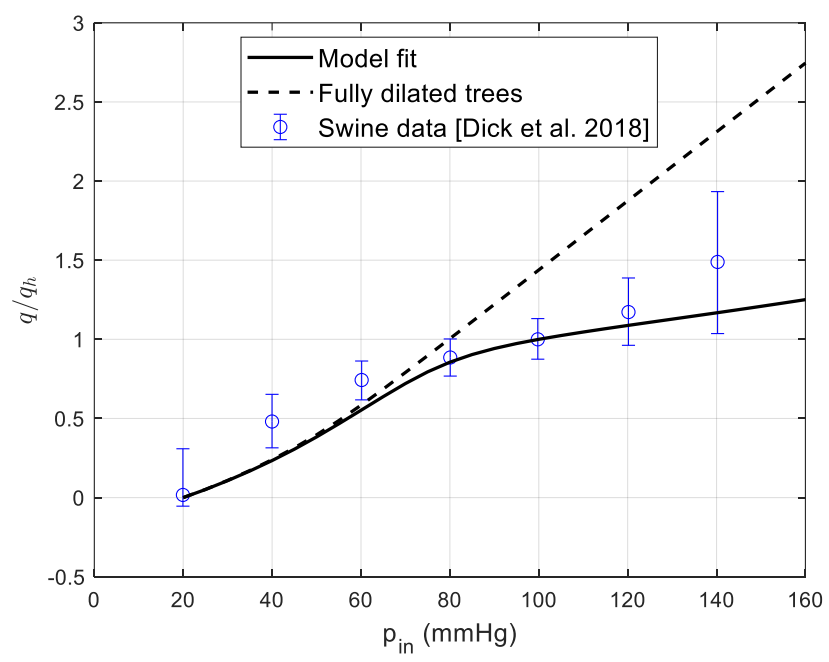

Figure 9. Pressure-flow autoregulation in combined trees compared to the collected experimental data in (21). The dashed line shows the pressure-flow relation when the entire tree is fully vasodilated. In the low coronary pressure range $p_{i n}<$ $60 \mathrm{mmHg}$, the autoregulatory and fully dilated responses are almost identical. $q_{h}$ is the baseline flow rate with coronary pressure $100 \mathrm{mmHg}$.

Figure 10 shows SMC activation level $(A)$, pressure-dependent stress $S_{p}$ (Eq. 3), and the SMC active tension $S$ (Eq. 2) as a function of coronary pressure. Our results indicate that the activation levels in the intermediate and small arterioles increase monotonically with coronary pressure (Figs. 10-A and B). Conversely, the activation level remains relatively constant in the small arteries and large arterioles over the entire pressure range Fig. 10. The pressure-dependent stress $S_{p}$ follows the same increasing trend with pressure in all the vessels in subepicardial tree (Figs. 10-C). However, this pressure-dependent stress is zero at low coronary pressure range in the subendocardium, since a large intramyocardial pressure in this layer ( $p_{i m}=47 \mathrm{mmHg}$, cf. Section 2.3) causes a negative transmural pressure throughout the tree when the inlet coronary pressure is small $\left(p_{i n}<47 \mathrm{mmHg}\right)$ (Fig. 10-D).

Active stress $S$ is determined by the multiplication of the activation level and pressure-dependent stress $S_{p}$ (Eq. 2). In low coronary pressures $p_{\text {in }}<50 \mathrm{mmHg}$, subendocardial vessels do not develop active stress $S$. Starting at pressure $\sim 50 \mathrm{mmHg}$ the active SMC stress increases in the subendocardial tree with the coronary pressure (Fig. 10-F). In subepicardial arteries and large arterioles, the active SMC stress $S$ is close to zero at $p_{\text {in }}=20 \mathrm{mmHg}$ but increases with coronary pressure. Conversely, it remains close to zero for small and intermediate arterioles (Fig. 10-E) for coronary pressures below $\sim 50 \mathrm{mmHg}$. 
bioRxiv preprint doi: https://doi.org/10.1101/2020.09.21.304030; this version posted September 22, 2020. The copyright holder for this preprint (which was not certified by peer review) is the author/funder, who has granted bioRxiv a license to display the preprint in perpetuity. It is made available under aCC-BY-ND 4.0 International license.

A

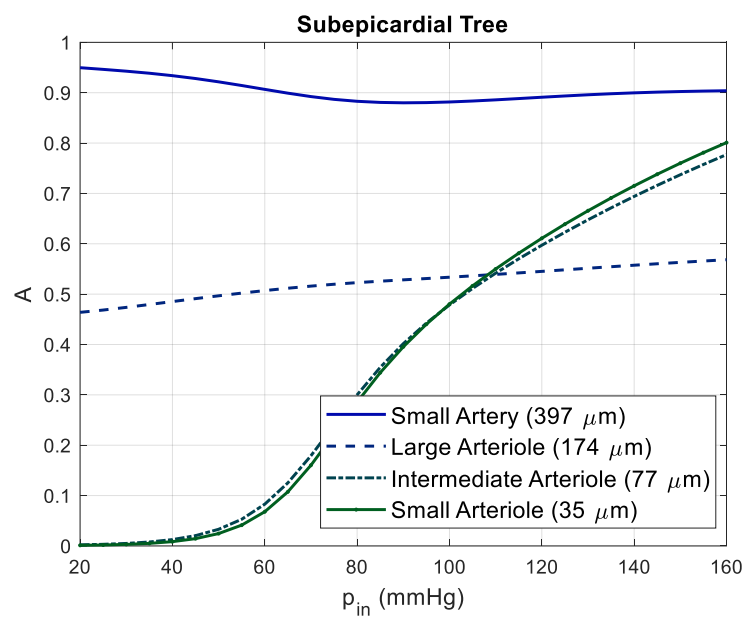

$\mathrm{C}$

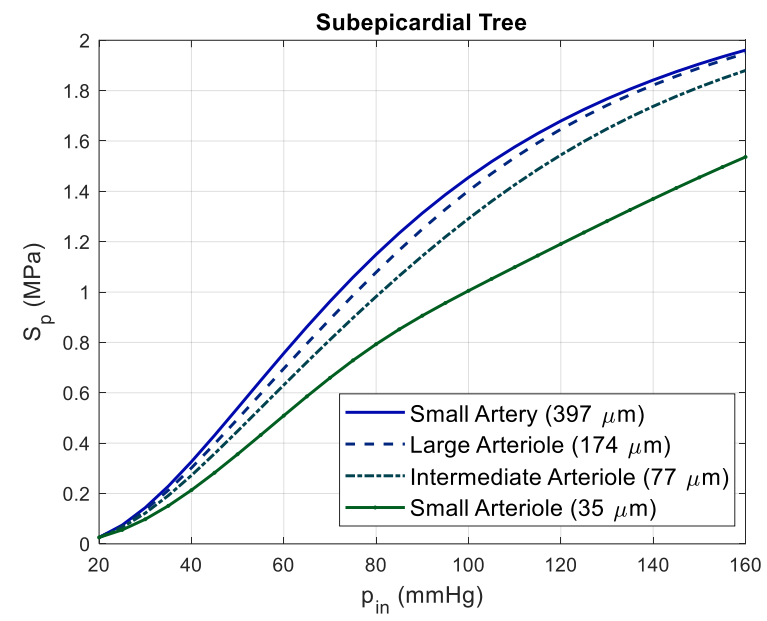

E

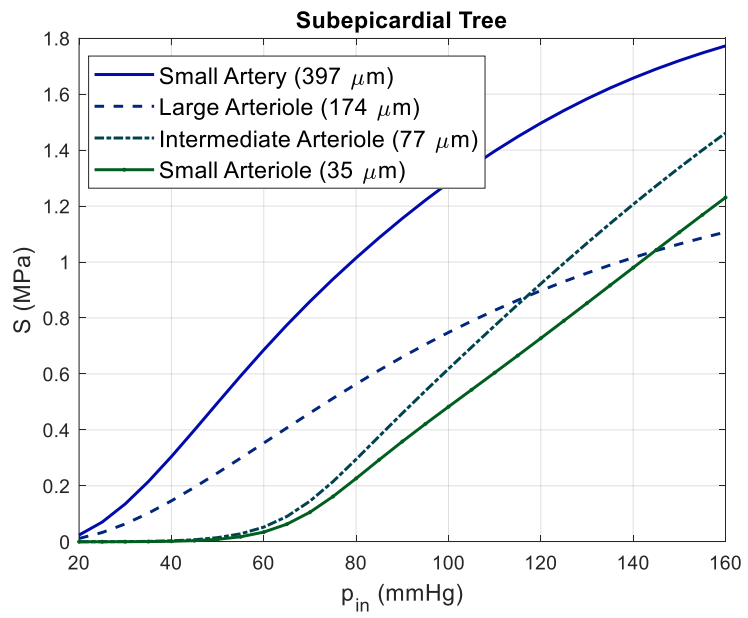

B

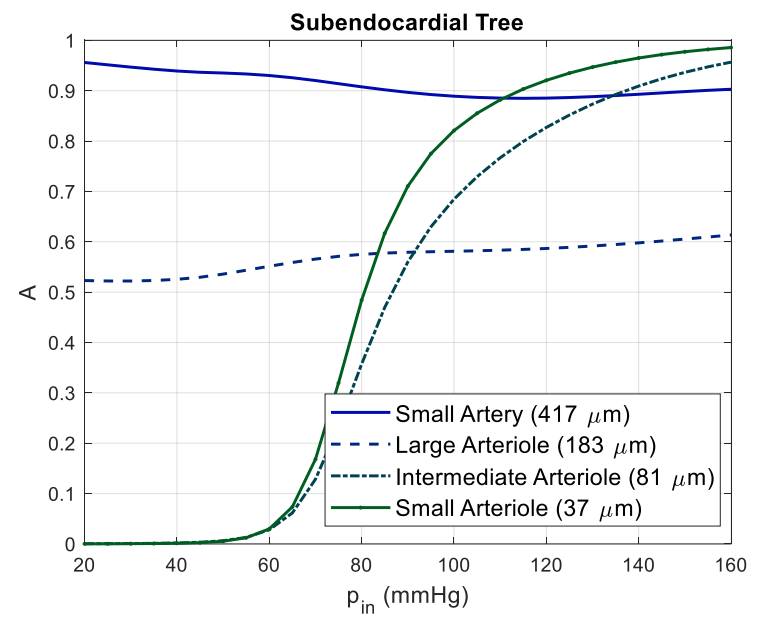

$\mathrm{D}$

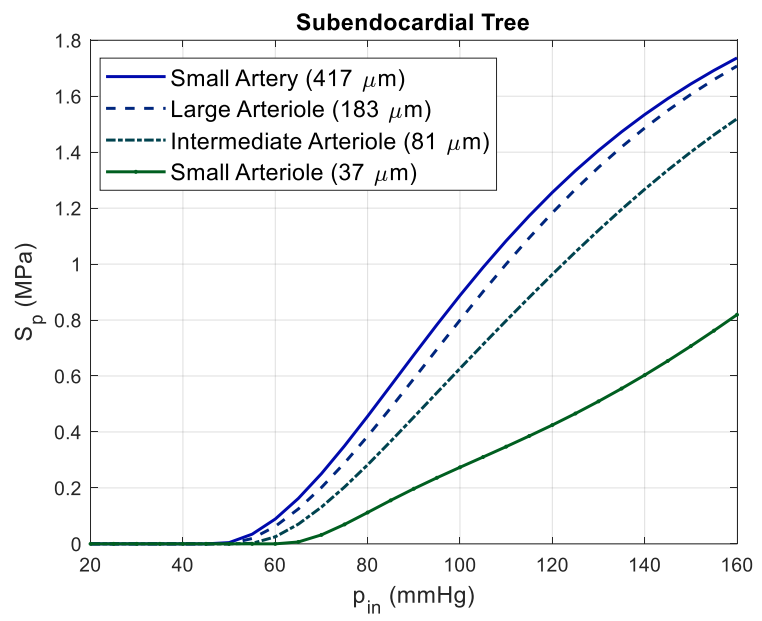

$\mathrm{F}$

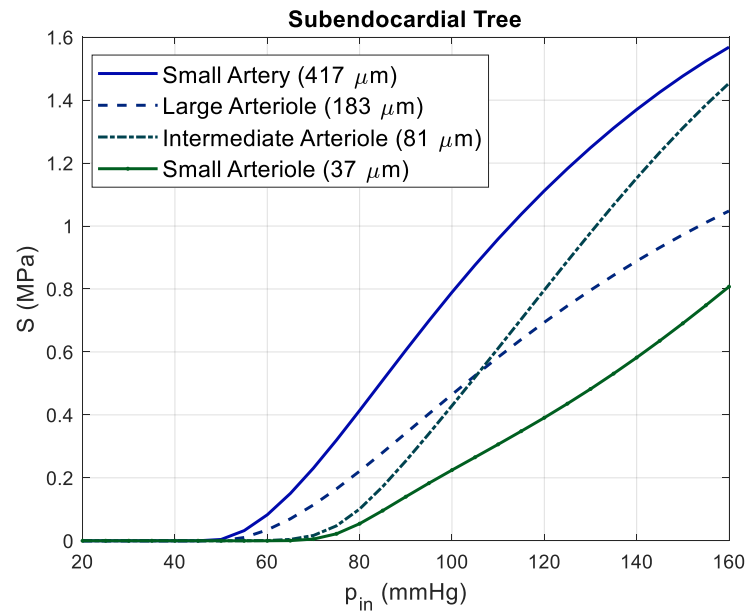

Figure 10. A \& B: Activation as a function of the pressure at the inlet of the myocardial arterial. C \& D: Pressuredependent active stress $S_{p}$. E \& F: Active SMC stress $S$. Curves are given for all vessel types (small arteries, large, intermediate, and small arterioles) and both vessel tress: subepicardial (left column), and subendocardial (right column). 


\subsubsection{Autoregulation responses for diameter, wall shear stress, and pressure}

Figure 11 shows the variations of normalized diameters $\left(D / D_{h}\right)$, normalized wall shear stresses $\left(\tau / \tau_{h}\right)$, and normalized luminal pressure $\left(p / p_{h}\right)$, for four representative vessels in both the subepicardium and subendocardium trees as a function of the coronary pressure. Mediated by the autoregulatory mechanisms (myogenic, metabolic, and shear-dependent), blood vessel diameters $D$ show a non-monotonic response to increase in coronary pressure in $20-160 \mathrm{mmHg}$ range. Such behavior has also been observed in autoregulation of other circulatory systems such as the mesenteric circulation (15). Wall shear stress, however, presents distinct response patterns for the intermediate and small arterioles compared to the small arteries and large arterioles: For the intermediate and small arterioles, the wall shear stress increases monotonically with pressure. However, the wall shear stress remains near its homeostatic value in the $p_{\text {in }}$ $=80-120 \mathrm{mmHg}$ range for small arteries and large arterioles in the subepicardial layer (Fig. 11-C) and in the $p_{\text {in }}=100-140 \mathrm{mmHg}$ range for small arteries and large arterioles in the subendocardial layer (Fig. 11D). Pressure in both coronary trees monotonically increases with the coronary pressure (Figs. 11-E and F).

A

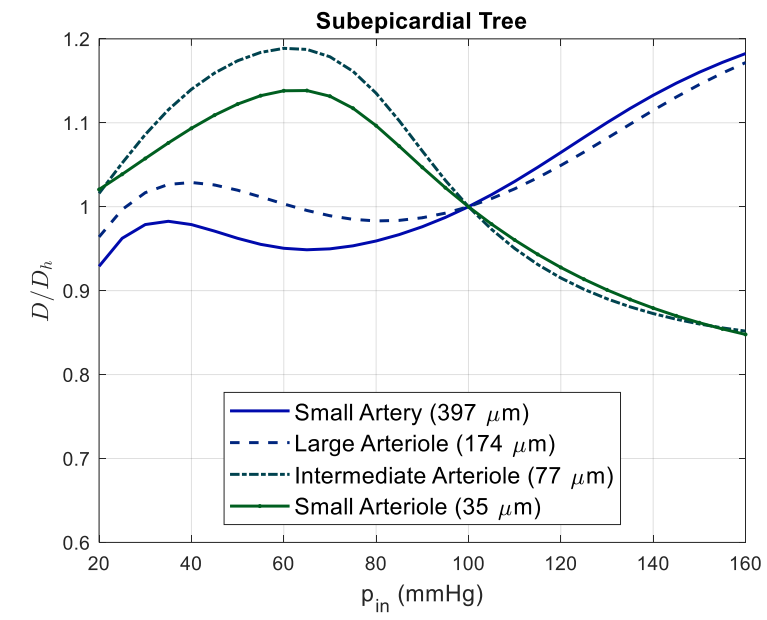

$\mathrm{C}$

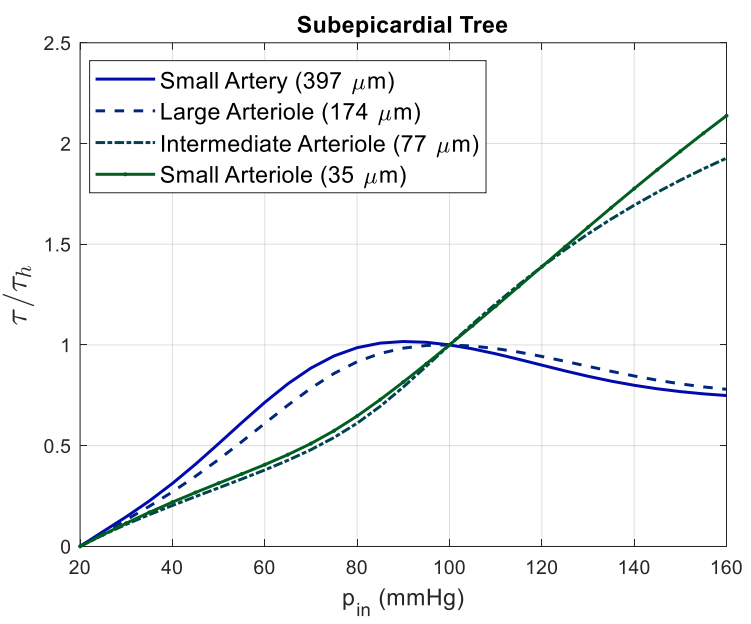

B

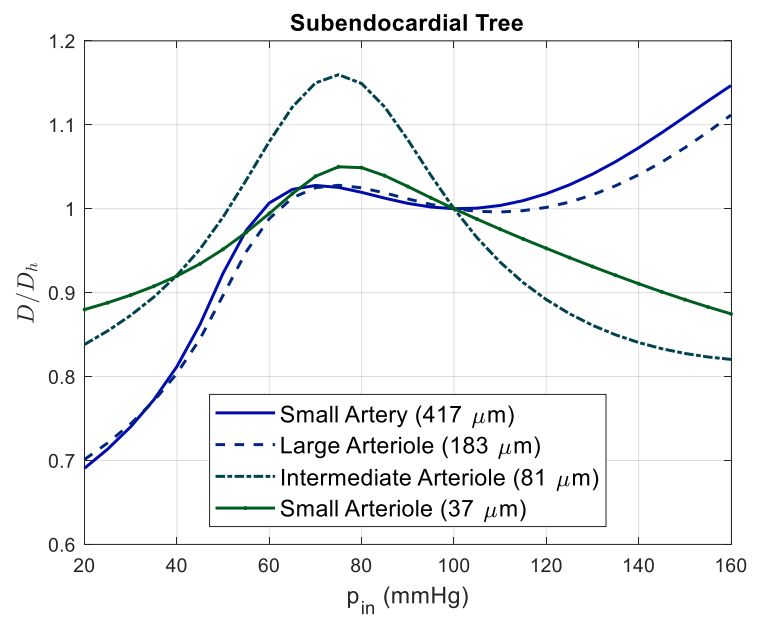

$\mathrm{D}$

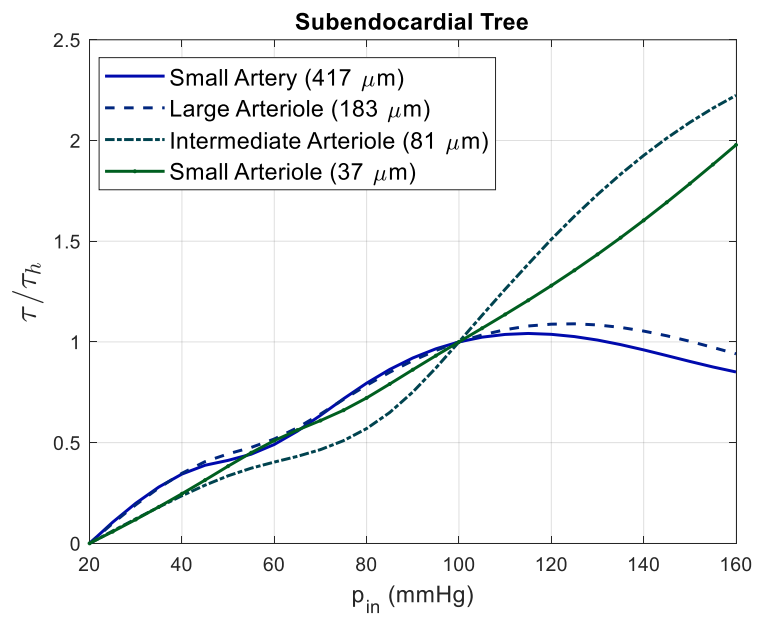


$\mathrm{E}$

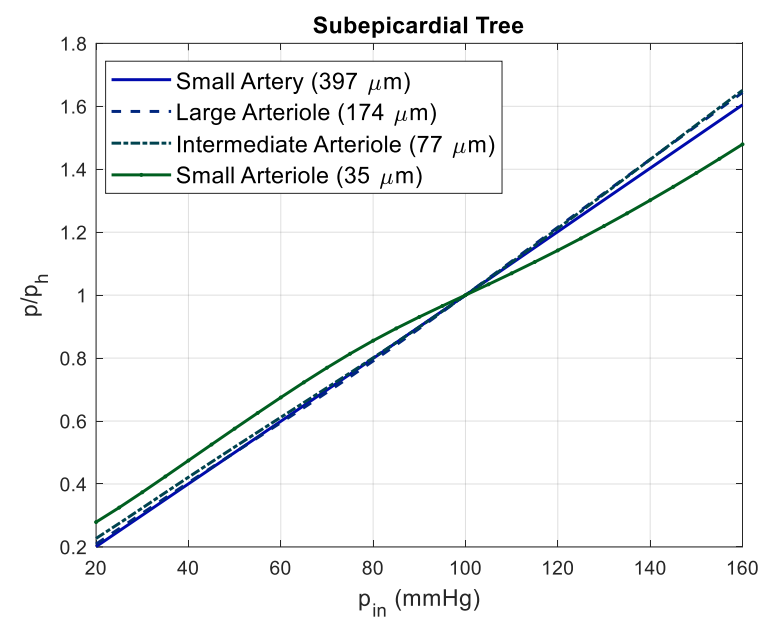

$\mathrm{F}$

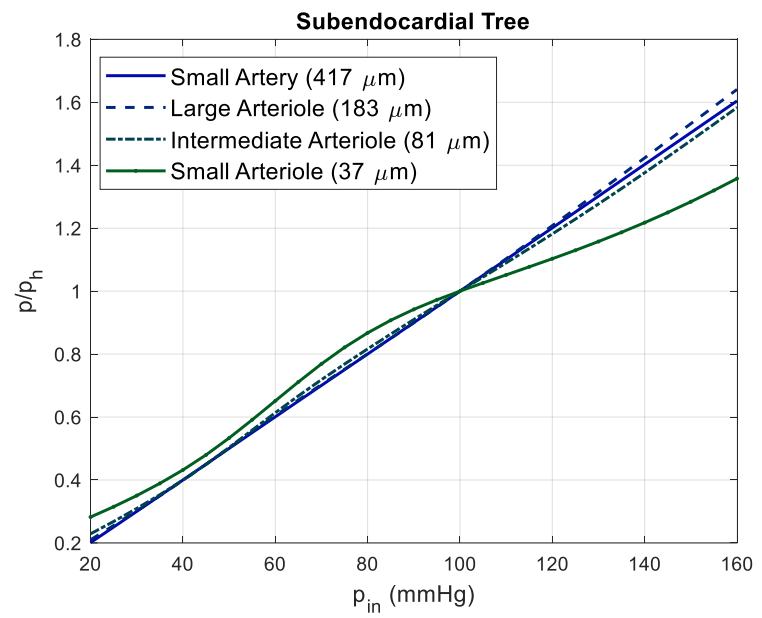

Figure 11. Normalized diameter $D / D_{h}$, wall shear stress $\tau / \tau_{h}$, and luminal pressure $\left(p / p_{h}\right)$ in four representative vessels as a function of the inlet coronary pressure. Vessel diameters $D$ show a non-monotonic response to increases in coronary pressure. Some degree of regulation of wall shear stress in response to moderate changes in coronary pressure was predicted in small arteries and large arterioles in both layers. Pressure in vessels of both coronary trees increase as a function of the coronary pressure

Figure 12 shows the predictions of our model for the subendocardial to subepicardial flow ratio (ENDO/EPI) over the 20-150 $\mathrm{mmHg}$ pressure range. ENDO/EPI blood flow ratios have been reported between 1.09 to 1.49 across different species (22). Our results show that in low coronary pressures ( $p_{\text {in }}<57$ $\mathrm{mmHg}$ ), the ENDO/EPI ratio falls below one and they qualitatively match experimental data in the literature. For instance, Ball and Bache (11) reported a 50-60\% decrease in the ENDO/EPI ratio in canine left ventricle as a result of mild to severe obstructions in large coronary arteries. Furthermore, Canty (14) indicated a redistribution of blood towards subepicardial tree $(\mathrm{ENDO} / \mathrm{EPI}<1)$ for coronary pressures lower than $\sim 33 \mathrm{mmHg}$.

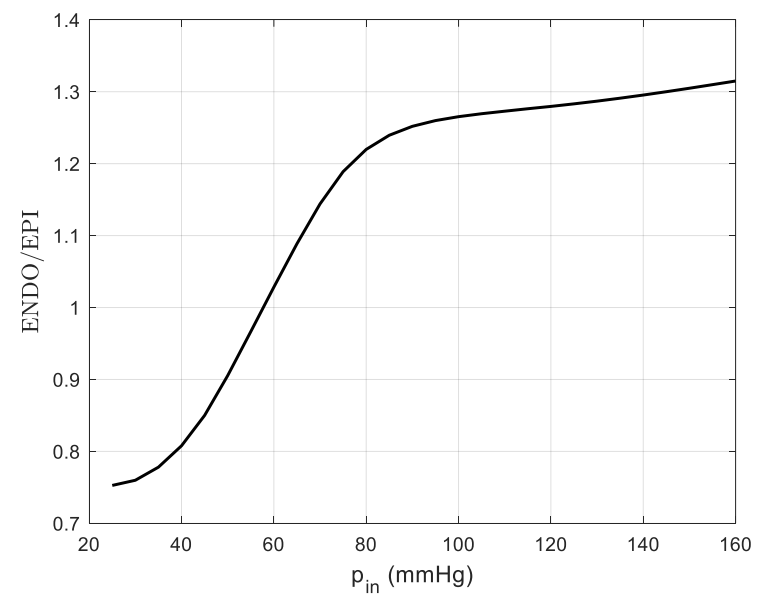

Figure 12. Transmural distribution of the blood flow during pressure-flow autoregulation. A regulation of the ENDO/EPI is predicted when the coronary pressure is $80-160 \mathrm{mmHg}$. In contrast, the ENDO/EPI drops significantly as the coronary pressure is reduced below $80 \mathrm{mmHg}$. 


\subsection{Model application: Response to drops in epicardial pressure}

Figure 13 shows the predictions of diameter changes in our model for each coronary tree due to mild $\left(p_{\text {in }}=59 \mathrm{mmHg}\right.$ ) and severe $\left(p_{\text {in }}=38 \mathrm{mmHg}\right.$ ) reductions in coronary perfusion pressure, compared with experimental data (45) on epicardial vessels at the same pressures. Although the pressure reductions across stenoses are triggered by different levels of epicardial occlusion, our choices of inlet pressures are motivated by the experimental study by Kanatsuka et al. (45). For mildly reduced coronary pressures, the subepicardial arterioles $\left(D_{h}<190 \mu \mathrm{m}\right)$ dilated, indicating a clear autoregulation response. Conversely, the subepicardial arteries remained near their baseline values, showing a mild vasoconstriction (Fig. 13-A). Our results for the subendocardial vessels did not reveal substantial changes in diameter relative to their baseline values for the entire tree.

A

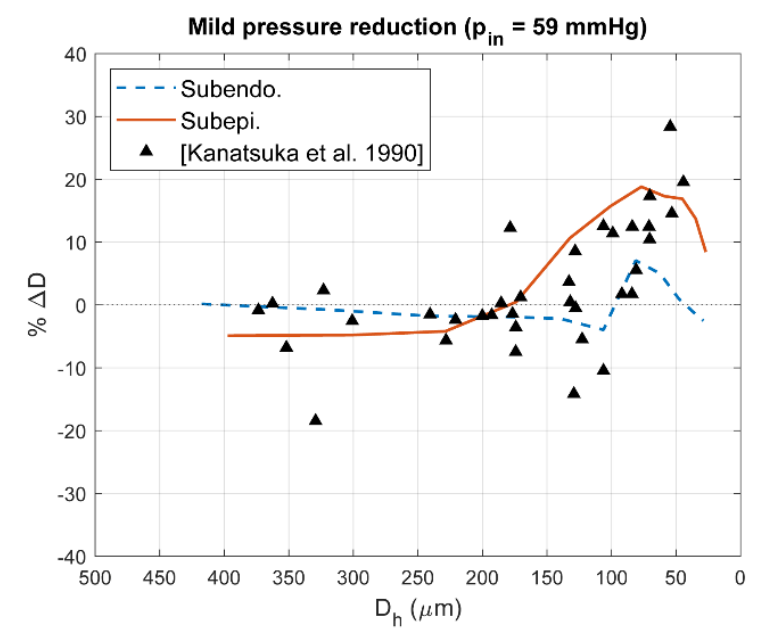

B

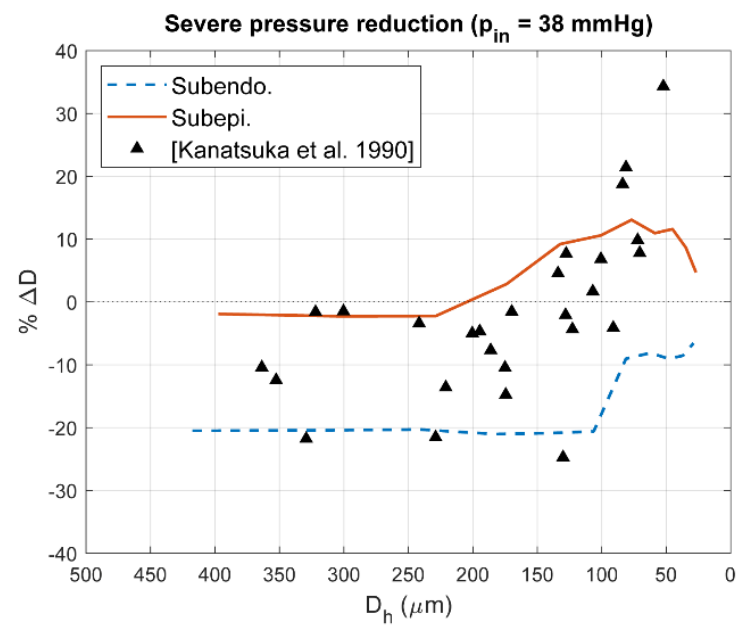

Figure 13. Effects of mild and severe pressure reduction on coronary arterial tree compared with experimental observations on canine epicardial vessels (45).

For a severe reduction in perfusion pressure, however, subepicardial vessels dilated in a similar fashion as in the mild reduction in pressure, while all subendocardial vessels constricted (Fig. 13-B). This subendocardial tree constriction is likely due to the large intramyocardial pressures $\left(p_{i m}=47 \mathrm{mmHg}\right.$ ), which in combination with the small luminal pressure $(38 \mathrm{mmHg}>p>20 \mathrm{mmHg}$ ), results in a negative transmural pressure and therefore in vasoconstriction. The dilation of the subepicardial tree and constriction of the subendocardial tree explains the ENDO/EPI flow redistribution in low coronary pressures (Fig. 13).

\subsection{Model application: Effects of adenosine and L-NAME infusion}

We further demonstrated our model by simulating responses of the coronary trees to two different pharmacological agents. Figure 14 shows the model results together with experimental data (41) for diameter changes in response to intracoronary adenosine and L-NAME infusion.

Adenosine is a metabolic vasodilator which binds to purinergic receptors and causes SMC relaxation mostly in the intermediate and small coronary arterioles (55). In this work, adenosine administration was modeled by setting the activation in intermediate and small arterioles $\left(D_{h} \leq 100 \mu \mathrm{m}\right)$ to zero $(A=0)$. Figure 14-A shows that adenosine infusion leads to up to $25 \%$ increase in the diameter of these arterioles, while the diameters of small arteries and large arterioles remain close to their baseline. 
L-NAME is an endothelial NO synthesis inhibitor (51). Based on the observations in the study by Jones et al. (41), L-NAME led to full myogenic constriction of small arteries and large arterioles $\left(D_{h}>100 \mu \mathrm{m}\right)$. However, they reported that although the experimental data showed both constrictive and dilatory responses in intermediate and small arterioles $\left(D_{h} \leq 100 \mu \mathrm{m}\right)$, most of the arterioles were dilated. In addition, they observed that administering adenosine to L-NAME infused hearts did not further affect the dilated arterioles, indicating that these arterioles were likely fully dilated in response to L-NAME. Therefore, we set $A=1$ for small arteries and large arterioles and $A=0$ for intermediate and small arterioles. Our results revealed small arteries vasoconstriction in both trees and a higher degree of vasoconstriction in the large subepicardial arterioles compared to their subendocardial counterparts (Fig. 14-B). The latter can be due to a higher transmural pressure $p_{t m}$ leading to larger myogenic pressure-dependent stress (Eq. 3). Diameter changes in intermediate arterioles $(50<\mathrm{D} \leq 100 \mu \mathrm{m})$ were similar in both coronary trees. Lastly, the small subendocardial arterioles $(\mathrm{D} \leq 50 \mu \mathrm{m})$ of were less dilated compared to the small subepicardial arterioles due to the larger intramyocardial pressure.

We must note that a direct comparison between model results and the experimental data on canine epicardial vessels is not feasible. This is due to the conceptual separation between subendocardial and subepicardial trees in our model, a separation not possible to establish in the experimental data. Furthermore, the experimental data in Figs. 13 and 14 were acquired in dogs, whereas our model had been calibrated using porcine data. Nevertheless, our model managed to capture the overall responses to changes in perfusion pressure and administration of adenosine and L-NAME.

A

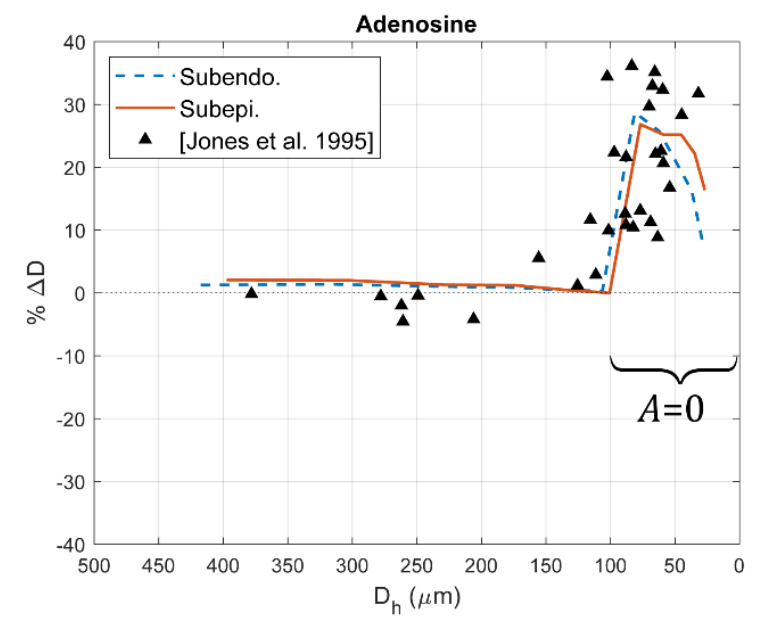

B

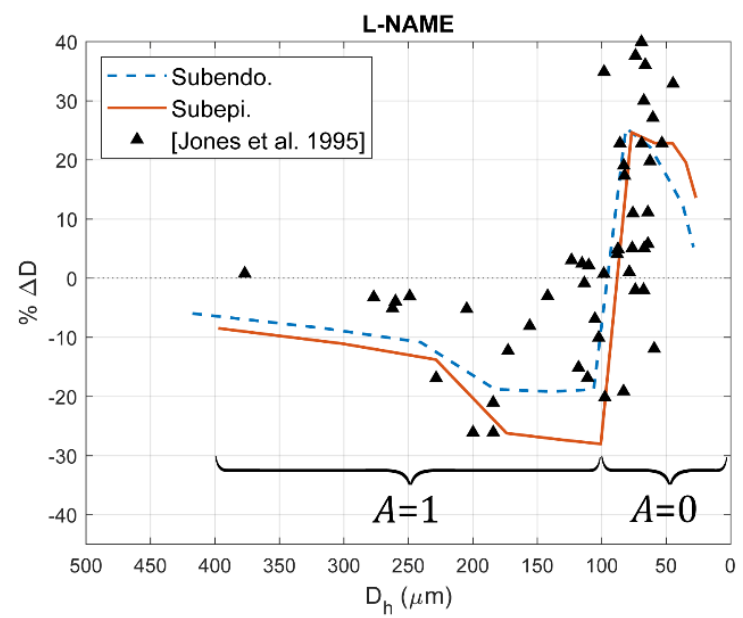

Figure 14. Effects of adenosine and L-NAME infusion on coronary trees compared with experimental canine data on epicardial microvascular response (41). Adenosine infusion was modeled by full dilation of small and intermediate arterioles $\boldsymbol{A}=\mathbf{0}$. L-NAME infusion was modeled by full dilation of small and intermediate arterioles $\boldsymbol{A}=\mathbf{0}$ and full myogenic constriction of large arterioles and small arteries $A=1$.

\section{Discussion}

The coronary circulation manifests a complex dynamic response to changes in perfusion pressure over multiple timescales. These timescales span from autoregulatory variations in active SMC tension, taking place within minutes, to slower changes in vessel microstructure (83) or vascular networks driven by angiogenesis or rarefaction over the course weeks to months (70).

Recent computational modeling endeavors in coronary physiology focused on capturing key features of short-term coronary autoregulation using lumped-parameter models $(18,66,89)$. However, since these 
approaches lack structurally-motivated models for vascular tissue, they are not equipped for predicting long-term pathophysiologic responses of the coronary circulation. To address this need, this work aimed to produce a unified computational approach that could integrate vascular responses at the short time scales (e.g., autoregulation) as well as long time scales (e.g. vascular growth and remodeling) while including morphometric, hemodynamics and structural data. This design goal resulted in the adoption of a structurally-motivated modeling framework, widely used by the tissue growth and remodeling community, such as the constrained mixture theory. The constrained mixture enables integration of microstructural properties and cellular level functions within the vessel wall in a nonlinear continuum mechanics framework. The proposed autoregulation model was developed in three stages (Fig. 1). The main findings of each of the three stages of the model is discussed next.

1. Constrained mixture model parameter estimation: The parameters of the constrained mixture model were estimated using passive and myogenic pressure-diameter data (55). These parameters included passive and active material properties, constituent pre-stretches, and their mass fractions. Experimental observations have shown that in the physiological condition and in the presence of in-vivo levels of active SMC tension, the extracellular matrix components in the arteriolar wall are wavy and are likely under a state of compression (58). This observation was captured in our parameter estimation which rendered a collagen fiber pre-stretch in the small arterioles smaller than 1 (Table 4). Another determining factor in the in-vivo structure of coronary vessels is their myocardial location. The significant intramyocardial pressure on subendocardial vessels $(47 \mathrm{mmHg}$ ) seems to compress collagen fibers even in large and intermediate arterioles (Table 4). Although the constituent pre-stretches are difficult to measure experimentally (37), they play a crucial role in determining the passive behavior of the vessel wall in its operating transmural pressure.

Furthermore, our model estimated a reduction in SMC mass fraction in the small arterioles relative to the large and intermediate arterioles (Fig. 4). This difference in estimated mass fractions seems to be due to the larger myogenic response in large and intermediate arterioles relative to small arterioles. A microscopic analysis of the mechanical structure of rabbit arterioles showed that the amount of SMCs gradually decreases from arterioles of $100 \mu \mathrm{m}$ to $30 \mu \mathrm{m}$ (75). Nevertheless, quantification of the mass fractions in both proximal and distal coronary arteries is essential for the mathematical modeling of functional (shortterm) and structural (long-term) vascular adaptations in pathophysiological conditions (34).

Our sensitivity analysis showed that our model results are the least sensitive to the passive material parameters of elastin $\left(c_{1}\right)$ and SMCs $\left(c_{4}\right.$ and $c_{5}$ ) (Table 4). Given that elastin is the main load bearing constituent at low pressures (88), the scarcity of pressure-diameter data in low pressure ranges $(<20 \mathrm{mmHg})$ may contribute to this insensitivity. In addition, the passive response of SMCs may not be distinguishable from that of collagen fibers since they both contribute to the exponential stiffening of the passive pressurediameter relations.

2. Definition of morphometry, structure, and hemodynamics in coronary trees: In the second stage of the model development, we used a homeostatic optimization to generate two symmetric coronary trees in subepicardial and subendocardial layers. This optimization (29) estimates the homeostatic morphometric (e.g, diameters), structural (e.g, thicknesses), and hemodynamics (e.g., wall shear stress) characteristics of a vascular tree by minimizing the metabolic and viscous energy dissipation under the constraint of mechanical equilibrium.

Two quantities were optimized in each vessel segment: 1) the homeostatic diameter and, 2) the total mass of wall constituents (elastin, collagen, and SMCs). Figure 6-8 summarize the estimated homeostatic 
characteristics of the coronary trees. Our model predicted a consistent diameter exponent (2.5-2.75) compared to experimental data (Fig. 6-C). In particular, several studies on the morphometry of vascular trees have shown a varying diameter exponent $\xi$ between 2 in larger vessels to near 3 in terminal arterioles $(47,60,62,79)$. Arts and Reneman (7) studied scaling laws in canine coronary vasculature and obtained an exponent of 2.55 for $\sim 400 \mu \mathrm{m}$ diameter vessels. Suwa and colleagues (79) obtained an exponent of 2.7 by analyzing different vascular beds in several organs of the human body. Furthermore, Kassab and Fung (47) obtained an exponent of 2.73 for arterioles smaller than $50 \mu \mathrm{m}$ in swine. In addition, our results for pressure, wall shear stress, and circumferential wall stress also showed reasonable agreement with experimental data as highlighted in Fig. 6. We must note that the inlet pressure for both subendocardial and subepicardial trees were assumed $100 \mathrm{mmHg}$ based on the study by Mittal et al. (62) that showed the pressure does not significantly drop in vessels of $100-1000 \mu \mathrm{m}$. The predicted homeostatic activation level $A_{h}$ seems to suggest that there exists a tradeoff between activation level and SMC mass fraction: a lower mass fraction of SMCs was associated with a higher activation for a given transmural pressure (Fig. 8).

3. Coronary autoregulation model: the proposed coronary autoregulation model was calibrated with experimental data on pressure-flow autoregulation. This is, to our knowledge, the first implementation of a constrained mixture theory to model coronary autoregulation. Our coronary autoregulation model is inspired by models previously proposed in the literature. For the myogenic control, we used the model proposed by (18), and for the shear-dependent and metabolic controls we used the activation model proposed by (15). This formulation was motivated by the ex-vivo experimental measurements in (55) where the transmural pressure controls the myogenic response. Alternatively, other modeling studies have assumed that the circumferential wall stress $\left(p_{t m} D / 2 H\right)$ sensed by the SMCs is the signal for the myogenic control mechanism $(38,87)$, based on earlier studies $(40)$. This theoretical discrepancy is circumvented in our model since the dependence of myogenic response on the vascular wall thickness $H$, vessel diameter $D$, and the transmural pressure $p_{t m}$ is implicitly integrated in the mechanical equilibrium equation. Furthermore, we designed our model such that we could separate the myogenic control from the other control mechanisms, to use the available myogenic pressure-diameter data. In addition, we formulated the shear-dependent and metabolic stimuli in terms of their deviation from a homeostatic value. This choice was motivated by previous applications of constrained mixture models in the study of long-term active SMC-mediated adaptations in cerebral vasospasms (10).

In this work, we showed that our model captures the essential pressure-flow coronary autoregulation over a pressure range of 70-140 $\mathrm{mmHg}$ (Fig. 9). However, our model showed larger deviations from experimental data for lower coronary pressures $(20-60 \mathrm{mmHg})$. Over that pressure range, our model closely followed the fully passive curve. This behavior is explained by the patterns of active SMC stress $S$ in Fig. 10-E and $\mathbf{F}$, which revealed nearly no active tension in any vessels of the subendocardial tree and active tension present only in small arteries and large arterioles of the subepicardial tree. This behavior suggests that the larger vessels of the subepicardial region do not play a significant role in coronary autoregulation.

Our model produced different patterns of vessel dilation and constriction depending on vessel size and location within the myocardium (Figs. 11-A and B). As pressure reduces from baseline, $p_{\text {in }}=100 \mathrm{mmHg}$, towards $p_{i n}=20 \mathrm{mmHg}$, small and intermediate subendocardial arterioles first dilate and then constrict (Figs. 11-B). The initial dilation of these vessels is driven by the autoregulatory response as seen by a decrease in activation $A$ and active SMC stress $S$ (Figs. 10-B and F). For coronary pressures $<60 \mathrm{mmHg}$ all the dilatory capacity in these vessels is exhausted $(A \sim 0)$, and the reduction in transmural pressure leads to passive constriction of these vessels. Similar trends can be observed for intermediate and small arterioles 
in the subepicardial layer (Fig. 11-A). These vessels, however, always remain dilated compared to their baseline value as the coronary pressure is decreased to $20 \mathrm{mmHg}$.

In addition, an analysis of the size-dependent heterogeneity of the autoregulatory response in the moderate coronary pressure range $(80-120 \mathrm{mmHg})$ reveals that the intermediate and small arterioles display more pronounced diameter changes in response to perturbations in coronary pressure, whereas larger vessels do not display similar variations (Figs. 11-A and B). Similar findings were reported in in-vivo epicardial coronary arterioles canine data (16), and feline and murine skeletal muscle arterioles $(32,61)$, where the proximal vessel diameters were almost irresponsive to pressure changes. The response of small arteries and large arterioles to changes in coronary pressure, however, is better understood in terms of the wall shear stress. Our results demonstrated that wall shear stress is regulated over a range of pressures $(80-120 \mathrm{mmHg})$ at the level of arteries and large arterioles (Figs. 10-C and D), whereas wall shear stress is not regulated in smaller vessels. Similar observations were reported in in-vivo experimental observations on canine coronary vessels (78).

We demonstrated our autoregulation model in two different application examples: 1) responses to drops in epicardial pressure and 2) responses to adenosine and L-NAME infusion. The first application example showed reasonable agreement between simulation and experimental data. Figure 12 shows that a severe reduction in pressure (e.g., severe stenosis) leads to a $20 \%$ diameter reduction of the proximal subendocardium vessels, rendering this layer susceptible to ischemia (4). This vulnerability was also evident in the redistribution of flow towards subepicardial layer in the pressure reductions $<60 \mathrm{mmHg}$ as shown in Fig. 12. Finally, our model was able to capture the experimental trends following adenosine and L-NAME infusion reported in (41) (Fig. 14). These results demonstrate that our in-silico model could successfully integrate experimental data on morphometry and structure and then reproduce key patterns of coronary autoregulation.

The present study has several limitations. First, the experimental measurements delineating the pressurediameter relationships of myocardial coronary vessels are scarce. We used four available sets of pressurediameter data for a wide range of subepicardial and subendocardial vessels from $400 \mu \mathrm{m}$ to $20 \mu \mathrm{m}$. More pressure-diameter data in the coronary arterioles will enhance the accuracy and predictive capabilities of the model. Although this limitation may not be influential in low coronary pressure ranges due to small activation, it may affect the total flow in high coronary pressures. In addition, the results of our constrained mixture model parameter estimation show a discrepancy with the myogenic pressure-diameter data in the small arterioles. In this work, coronary vascular networks were idealized to bifurcating trees with 11 generations, whereas a realistic reconstruction of the coronary network could impact the local hemodynamics and autoregulatory responses. Moreover, there is no cross-talk between the subendocardial and subepicardial trees in our model, whereas in reality the two layers of the coronary tree compete for flow in situations of low perfusion pressure. Our model was constructed based on steady state hemodynamics. However, hemodynamics in the coronary vessels are pulsatile. This pulsatility likely influences the homeostatic conditions of the wall constituents. Lastly, understanding the mechanisms involved in the coronary autoregulation, especially the role of metabolic control, has been a largely debated issue in coronary physiology (49). In this study we simulated coronary autoregulation using the three main mechanisms and their range of influence as defined in previous modeling studies. Our model, however, could be further refined with improvements in our understanding of the interplay between the different mechanisms of coronary autoregulation. 


\section{Conclusion}

The study of physiology and pathophysiology in coronary circulation benefits from accurate mathematical models that can account for both the microstructure and physiological function of arterial networks. In this study, we presented a structurally-motivated coronary autoregulation model that uses a nonlinear continuum mechanics approach to account for the morphometry and vessel wall composition in two idealized coronary trees. Literature data were used to calibrate and test our model. With some modifications, this model can be applied to morphometry-based coronary trees instead of idealized symmetric trees. Finally, since our model is based on constrained mixture theory, it could be expanded to also study long-term growth and remodeling in the coronary circulation in response to hypertension, atherosclerosis, etc.

\section{ACKNOWLEDGEMENT}

This study was supported by the NIH U01-HL135842 and R01-HL139813. We would like to also acknowledge Prof. Lik Chuan Lee and Dr. Vasilina Filonova for their valuable insights.

\section{Appendix}

\section{A. Mechanics of a single artery}

A single segment of the arterial tree is considered as a thin-walled cylindrical tube composed of three main load-bearing constituents: elastin $(e)$, collagen $(c)$, and smooth muscle cells (SMCs; $m$ ). First, we only consider the passive response of constituents. Each constituent is assumed to separately contribute to the strain energy density:

$$
w=w^{e}\left(\boldsymbol{F}_{n}^{e}\right)+w^{m}\left(\boldsymbol{F}_{n}^{m}\right)+w^{c}\left(\boldsymbol{F}_{n}^{c}\right)
$$

where $\boldsymbol{F}_{n}^{i}$ is the deformation gradient of each constituent $i(i \in\{e, m, c\})$ corresponding to its map from a stress-free configuration to the overall homeostatic configuration. We define this deformation gradient as $\boldsymbol{F}_{n}^{i}=\boldsymbol{F G}^{i}$, where $\boldsymbol{F}$ represents the deformation gradient of the mixture mapping from its reference configuration to the homeostatic configuration, and $\boldsymbol{G}^{i}$ is a pre-stretch for each constituent mapping each constituent from its distinct stress-free configuration to the reference configuration (9). In particular, the pre-stretch mapping for elastin can be expressed as

$$
\boldsymbol{G}^{e}=\left(\begin{array}{ccc}
G_{r}^{e} & 0 & 0 \\
0 & G_{\theta}^{e} & 0 \\
0 & 0 & G_{z}^{e}
\end{array}\right)
$$

where $G_{\theta}^{e}$ and $G_{z}^{e}$ are pre-stretches associated with circumferential and axial directions, and $G_{r}^{e}=1 / G_{\theta}^{e} G_{z}^{e}$.

Similarly, for collagen fibers and SMCs, $\boldsymbol{M}^{\boldsymbol{i}}, i \in\{k, m\}$ is defined as the unit vector in the direction of the collagen fiber $(k)$ or SMCs. The pre-stretch mappings for collagen and smooth muscle cells are given as

$$
\boldsymbol{G}^{\boldsymbol{k}}=G_{h}^{c} \boldsymbol{M}^{\boldsymbol{k}} \otimes \boldsymbol{M}^{\boldsymbol{k}}, \boldsymbol{G}^{\boldsymbol{m}}=G_{h}^{m} \boldsymbol{M}^{\boldsymbol{m}} \otimes \boldsymbol{M}^{\boldsymbol{m}},
$$

where the pre-stretches $G_{h}^{c}$ and $G_{h}^{m}$ are also called homeostatic stretches, the stretches of the constituents when they are produced. We should note that in the previous applications of growth and remodeling, the pre-stretches were assumed to be constant for a single vessel. In our generalization of the framework to a vascular tree, we account for the variation of pre-stretches across the generations of vessels. Nevertheless, the pre-stretch implies that the homeostatic state in an individual vessel is associated with a constant homeostatic stress for the constituents of the vessel wall. 
The orientation of collagen fibers and smooth muscles with respect to the axial direction in their reference configuration, defined by angle $\gamma^{k}$, can be written as

$$
\boldsymbol{M}^{\boldsymbol{k}}=\cos \left(\gamma^{k}\right) \boldsymbol{e}_{\boldsymbol{Z}}+\sin \left(\gamma^{k}\right) \boldsymbol{e}_{\boldsymbol{\theta}}, \boldsymbol{M}^{\boldsymbol{m}}=\boldsymbol{e}_{\boldsymbol{\theta}}
$$

For modeling the extension and inflation of a thin wall model, $\boldsymbol{F}$ is considered as $\boldsymbol{F}=\operatorname{diag}\left[\lambda_{r}, \lambda_{\theta}, \lambda_{z}\right]$. The stretches of constituent $i, \lambda^{i}, i \in\{e, k, m\}$, are expressed in terms of the pre-stretches using $\boldsymbol{F}^{i}=\boldsymbol{F G}^{\boldsymbol{i}}$

$$
\lambda_{\theta}^{e}=G_{\theta}^{e} \lambda_{\theta}, \lambda_{z}^{e}=G_{z}^{e} \lambda_{z}, \lambda^{k}=G_{h}^{c} \sqrt{\lambda_{\theta}^{2} \sin ^{2} \gamma^{k}+\lambda_{z}^{2} \cos ^{2} \gamma^{k}}, \lambda^{m}=G_{h}^{m} \lambda_{\theta}
$$

The incompressibility of the wall material is imposed by assuming an isochoric motion (i.e., $\operatorname{det}(\boldsymbol{F})=1$ ), and thus $\lambda_{r}=1 / \lambda_{\theta} \lambda_{z}$. Using the membrane theory (36), the passive membrane Cauchy stress (force per deformed length) can be written as

$$
\boldsymbol{T}_{p a s}=\frac{1}{J_{2 D}} \boldsymbol{F} \frac{\partial w}{\partial \boldsymbol{F}^{\boldsymbol{T}}} \Rightarrow T_{\theta \theta}=\frac{1}{\lambda_{z}} \frac{\partial w}{\partial \lambda_{\theta}}, \text { and } T_{z z}=\frac{1}{\lambda_{\theta}} \frac{\partial w}{\partial \lambda_{z}},
$$

where $J_{2 D}=\lambda_{\theta} \lambda_{z}$. The total strain energy per unit area can be written as

$$
w=M_{R}^{e} \Psi^{e}+\sum_{k} M_{R}^{k} \Psi^{k}+M_{R}^{m} \Psi^{m}
$$

where $M_{R}^{i}, i \in\{e, k, m\}$ is the mass of each constituent per unit reference area. Alternatively, the total strain energy per unit area can be written as

$$
w=M_{R}^{\text {total }}\left(v^{\mathrm{e}} \Psi^{e}+\sum_{k} v^{k} \Psi^{k}+v^{m} \Psi^{m}\right),
$$

where $v^{\mathrm{e}}, v^{k}$, and $v^{m}$ are mass fractions of elastin, collagen fiber families, and SMCs, respectively. In this work, four families of collagen fibers in circumferential, axial, and two diagonal directions were considered with mass fractions $v^{k}=(0.1,0.1,0.4,0.4) v^{c}$ where $v^{c}$ is the total collagen mass fraction (90). The total mass per unit area $M_{R}^{\text {total }}$ is the mass of load bearing constituents and can be computed via

$$
M_{R}^{\text {total }}=\left(1-\phi_{f}\right) \rho_{\text {wall }} H,
$$

where $\rho_{\text {wall }}$ is the density of the vascular wall, $\phi_{f}$ is the volume fraction of interstitial fluid, and $H$ is the thickness under homeostatic conditions.

A neo-Hookean model is employed for the passive elastin response and a Holzapfel exponential model is used for collagen fiber families and passive behavior of circumferentially oriented SMCs

$$
\begin{gathered}
\Psi^{e}=\frac{c_{1}}{2}\left\{\left(\lambda_{\theta}^{e}\right)^{2}+\left(\lambda_{z}^{e}\right)^{2}+\frac{1}{\left(\lambda_{\theta}^{e}\right)^{2}\left(\lambda_{z}^{e}\right)^{2}}-3\right\}, \\
\Psi^{k}\left(\lambda_{n}^{k}\right)=\frac{c_{2}}{4 c_{3}}\left\{\exp \left[c_{3}\left(\lambda_{n}^{k^{2}}-1\right)^{2}\right]-1\right\}, \\
\Psi^{m}\left(\lambda_{n}^{m}\right)=\frac{c_{4}}{4 c_{5}}\left\{\exp \left[c_{5}\left(\lambda_{n}^{m^{2}}-1\right)^{2}\right]-1\right\} .
\end{gathered}
$$


Where $c_{1}$ is the elastin material parameter, $c_{2}$ and $c_{3}$ are collagen material parameters, and $c_{4}$ and $c_{5}$ are passive SMC material parameters. To include the active tension of vascular SMCs, we use a potential function as given by (10)

$$
\Psi_{a c t}^{m}=\frac{S}{\rho_{\text {wall }}}\left\{\lambda_{\theta}+\frac{1}{3} \frac{\left(\lambda_{M}-\tilde{\lambda}\right)^{3}}{\left(\lambda_{M}-\lambda_{0}\right)^{2}}\right\},
$$

where $\lambda_{M}$ and $\lambda_{0}$ are stretches at which the active force generation is maximum and zero, respectively, and $\rho_{\text {wall }}$ is the vascular wall density. In addition, $\tilde{\lambda}$ is an active stretch of the SMCs in the circumferential direction, which can evolve by SMC remodeling over slow timescales (hours to days). In the current study, we focus on the short timescale adaptations (minutes), and thus, $\tilde{\lambda}$ is assumed to be the total circumferential stretch in the vessel $\left(\lambda_{\theta}\right)$. Active SMC stress $S$ is in general a function of the myogenic, shear-dependent, and metabolic controls in coronary vessels. Again, using membrane theory, $T_{a c t}=\frac{1}{\lambda_{z}} \frac{\partial M_{R}^{m} \psi_{a c t}^{m}}{\partial \lambda_{\theta}}$ the total tension in the artery can be written as

$$
\mathbf{T}=\mathbf{T}_{\text {pass }}+T_{a c t} \mathbf{e}_{\theta} \otimes \mathbf{e}_{\theta}
$$

Finally, for a thin walled cylinder with transmural pressure $p_{t m}$, the force equilibrium in the circumferential direction gives

$$
\frac{p_{t m} D}{2}=T_{\theta \theta}+T_{a c t}
$$

where $D$ is the vessel diameter and $p_{t m}$ is the transmural pressure of the artery.

We assume the reference configuration of the blood vessel in our continuum mechanics formulation is its homeostatic configuration. Therefore, by setting $\boldsymbol{F}=\boldsymbol{I}$ (i.e., $\lambda_{\theta}=\lambda_{z}=1$ ) in equations above, we can write

$$
\frac{p_{t m}^{h} D_{h}}{2}=T_{\theta \theta}^{h}+T_{a c t}^{h}
$$

where $T_{\theta \theta}^{h}$ and $T_{a c t}^{h}$ are homeostatic passive and active circumferential tensions, respectively, $p_{t m}^{h}$ is the homeostatic transmural pressure and $D_{h}$ is the homeostatic diameter of the vessel. The homeostatic passive tension $T_{\theta \theta}^{h}$ is thus determined by the material properties $\left(c_{1}-c_{5}\right)$, constituent prestretches $\left(G_{\theta}^{e}, G_{z}^{e}, G_{h}^{c}\right.$, and $G_{h}^{m}$ ) and their mass fractions $v^{i}$. In addition, the homeostatic active tension $T_{\theta \theta}^{h}$ is determined by active SMC parameters, $\lambda_{M}, \lambda_{0}$ and $S_{h}$. Accordingly, we can also compute the homeostatic stress in each constituent $\boldsymbol{\sigma}^{i}$ as a function of its material parameters, its homeostatic stretch $G^{i}$, and active SMC stress in case of SMCs.

\section{B. Parameter estimation}

The parameter estimations in sections 2.2 and 2.4 are performed by minimizing the following error function:

$$
E=\sqrt{\sum_{\text {experiment }}\left(\mathrm{y}^{\mathrm{th}}-\mathrm{y}^{\text {exp }}\right)^{2}}
$$

where $y^{\text {th }}$ and $y^{\text {exp }}$ are theoretical and experimental values of the quantity $y$. In section 2.2 , the quantity $y$ is the diameter of vessels in passive and myogenic responses, and in section 2.4, the flow in the coronary arteries $(q)$ over the flow when coronary pressure is $100 \mathrm{mmHg}$. Specific constraints for the optimization 
problem are stated in the text.

\section{Homeostatic optimization}

Originally introduced by Murray (64), and later extended by Taber (80) and Lindström et al. (56), the extended Murray's law states that the vessel wall composition and geometry strive to minimize the energy consumption. The homeostatic optimization framework proposed in (29) leverages this idea to identify the homeostatic characteristics of an arterial tree. Briefly, the total energy cost per unit length $C$ for an individual blood vessel can be written as

$$
C\left(M_{R}^{i}, R ; q\right)=C_{\text {blood }}+C_{\text {drag }}+C_{\text {wall }}=\alpha^{\text {blood }} \pi R^{2}+\frac{8 \mu q^{2}}{\rho_{\text {blood }} R^{4}}+\frac{2 \pi R}{\rho_{\text {wall }}} \sum_{i} \alpha^{i} M_{R}^{i},
$$

where $C_{\text {blood }}$ is the metabolic cost of sustaining blood in the vessels, $C_{\text {drag }}$ is the power needed to overcome viscous drag, and $C_{\text {wall }}$ is the metabolic cost of maintaining the constituents of the wall. In addition, $\alpha^{\text {blood }}$ and $\alpha^{i}$ are the metabolic energy costs of blood and wall constituents $(i \in\{e, k, m\}$, see Appendix A) per unit volume, $R(=D / 2)$ is the radius of the vessel, $q$ is the blood flow in the vessel, and $\mu, \rho_{\text {blood }}$, and $\rho_{\text {wall }}$ are the blood viscosity, blood density, vessel wall density, respectively. We must note that the metabolic cost of SMCs can be written as the summation of the metabolic cost of their maintenance $\alpha_{\text {maint }}^{m}$ and the metabolic cost of the active SMC stress $\alpha_{\text {active }}^{m}$.

$$
\alpha^{m}=\alpha_{\text {maint }}^{m}+\alpha_{\text {active }}^{m} S_{h}
$$

where $S_{h}$ is the homeostatic active SMC stress. This metabolic cost function has to be minimized with Eq. A.14 as the constraint in each segment. With regards to Appendix A, we can rewrite the mass of constituents in terms of their mass fractions as $v^{e}=M_{R}^{e} / M_{R}^{\text {total }}, v^{m}=M^{m} / M_{R}^{\text {total }}$, and $v^{k}=M_{R}^{k} / M_{R}^{\text {total }}$ with $v^{c}=$ $\sum v^{k}$. Using the mass fractions and considering that the arteries are in homeostatic state, we can write the mechanical equilibrium in each vessel as

$$
p_{t m} R=\frac{M_{R}^{\text {total }}}{\left(1-\phi_{f}\right) \rho_{\text {wall }}} \sum_{i} v^{i} \sigma_{\theta}^{i}
$$

where $\phi_{f}$ is the volume fraction of interstitial fluid (70\%) and $\sigma_{\theta}^{i}$ is the circumferentially acting part of the homeostatic stress tensor $\boldsymbol{\sigma}^{\boldsymbol{i}}$. Therefore, the energy cost of one vessel can be written in terms of diameter, with pressure and flow rate given from the hemodynamics as

$$
C\left(R ; q, p_{t m}\right)=\alpha^{\text {blood }} \pi R^{2}+\frac{8 \mu q^{2}}{\rho_{\text {blood }} R^{4}}+2 \pi\left(1-\phi_{f}\right) p_{t m} R^{2} \frac{\sum_{i} v^{i} \alpha^{i}}{\sum_{i} v^{i} \sigma_{\theta}^{i}},
$$

The total energy cost of all arteries can be added to compute the total cost for maintenance of an arterial tree

$$
C_{\text {total }}=\sum_{j} C_{j} L_{j}
$$

where $C_{j}$ is the energy cost (Eq. B.3) and $L_{j}$ is the length of segment $j$. The optimization requires the hemodynamics parameters such as $p_{\text {in }}, p_{\text {out }}$, and $q_{\text {total }}$ as constraints. In this study, we fix the pressures at the inlet and outlets and incorporate the flow rate as a penalty term to the cost function. 


$$
C_{\text {total }}=\sum_{j} C_{j} L_{j}+k\left|q_{1}-\bar{q}\right|^{2},
$$

where $q_{1}$ is the total flow in the tree, $\bar{q}$ is the approximated flow in the tree from hemodynamic data, and $k$ is a penalty coefficient. Once the radius of each vessel is determined by minimization of $C_{\text {total }}$, the thickness of each vessel can be computed using equations B.2 and A.10. As explained in Appendix A, the homeostatic stress tensor $\boldsymbol{\sigma}^{\boldsymbol{i}}$ for each constituent is a function of its material parameters, its homeostatic pre-stretches, and the active stress $S$ for SMCs. In this work, to find the homeostatic characteristics of the vessel wall in the tree, material parameters and pre-stretches are determined according to their diameter using the 4 types of vessels; small arteries, large, intermediate, and small arterioles. However, the SMC active stress $S$ is tuned for each individual vessel in the tree by changing SMC activation $A$ to fit the thickness to diameter ratio reported in Guo and Kassab (33). The parameters used for the homeostatic optimization are summarized in Table B1. thickness-to-diameter ratio with data in (33), as demonstrated in the results section.

Table B1. Parameters of the homeostatic optimization

\begin{tabular}{ccccc}
\hline Parameter & Description & Value & Unit & Reference \\
\hline $\begin{array}{c}\alpha^{c} \text { and } \\
\alpha_{\text {maint }}^{m}\end{array}$ & $\begin{array}{c}\text { Metabolic cost of collagen and } \\
\text { SMCs per unit volume }\end{array}$ & 1070 & $\mathrm{~W} / \mathrm{m}^{3}$ & $(68)$ \\
\hline$\alpha^{e}$ & $\begin{array}{c}\text { Metabolic cost of elastin per unit } \\
\text { volume }\end{array}$ & $\begin{array}{c}\text { (Only produced } \\
\text { at early ages.) }\end{array}$ & $\mathrm{W} / \mathrm{m}^{3}$ & $(56)$ \\
\hline$\alpha^{\text {blood }}$ & $\begin{array}{c}\text { Metabolic cost of blood per unit } \\
\text { volume }\end{array}$ & 51.7 & $\mathrm{~W} / \mathrm{m}^{3}$ & $(57)$ \\
\hline$\alpha_{\text {active }}^{m}$ & Metabolic cost of active SMC stress & 0.00872 & $1 / \mathrm{s}$ & $(68)$ \\
\hline $\begin{array}{l}\rho_{\text {wall }}, \\
\rho_{\text {blood }}\end{array}$ & Vessel and blood density & 1060 & $\mathrm{~kg} / \mathrm{m}^{3}$ & - \\
\hline
\end{tabular}

\section{Viscosity}

Blood viscosity in the systemic vasculature depends on the size of the vessel and the hematocrit level $\left(H_{D}\right)$. Particularly, the variation of viscosity is more pronounced as the arteries and arterioles become smaller. In our study, we prescribe the viscosity using the following in-vivo viscosity law given in (73)

$$
\begin{gathered}
C=\left(0.8+e^{-0.07 D}\right)\left(-1+\frac{1}{\left(1+10^{-11} D^{12}\right)}\right)+\frac{1}{\left(1+10^{-11} D^{12}\right)}, \\
h_{f}=\frac{\left(1-H_{D}\right)^{C}-1}{(1-0.45)^{C}-1} \\
\mu_{0.45}=6 e^{(-0.085 D)}+3.2-2.44 e^{-0.06 D^{0.645}} \\
\mu_{\text {vivo }}=\mu_{0}\left(1+h_{f}\left(\mu_{0.45}-1\right)\left(\frac{D}{D-1.1}\right)^{2}\right)\left(\frac{D}{D-1.1}\right)^{2},
\end{gathered}
$$

where $D$ is the diameter in micron $(2 R)$, and $\mu_{0}$ is the viscosity of the blood plasma taken to be 0.001 Pa.s. 


\section{References}

1. Aharinejad S, Schreiner W, Neumann F. Morphometry of human coronary arterial trees. Anat Rec 251: 50-59, 1998. doi: 10.1002/(SICI)1097-0185(199805)251:1<50::AID-AR9>3.0.CO;2-I.

2. Alford PW, Humphrey JD, Taber LA. Growth and remodeling in a thick-walled artery model: Effects of spatial variations in wall constituents. Biomech Model Mechanobiol 7: 245-262, 2008. doi: 10.1007/s10237-007-0101-2.

3. Algranati D, Kassab GS, Lanir Y. Mechanisms of myocardium-coronary vessel interaction. Am J Physiol Circ Physiol 298: H861-H873, 2010. doi: 10.1152/ajpheart.00925.2009.

4. Algranati D, Kassab GS, Lanir Y. Why is the subendocardium more vulnerable to ischemia? A new paradigm. Am J Physiol - Hear Circ Physiol 300, 2011. doi: 10.1152/ajpheart.00473.2010.

5. Arciero JC, Carlson BE, Secomb TW. Theoretical model of metabolic blood flow regulation: roles of ATP release by red blood cells and conducted responses. Am J Physiol Circ Physiol 295: H1562H1571, 2008. doi: 10.1152/ajpheart.00261.2008.

6. Arthurs CJ, Lau KD, Asrress KN, Redwood SR, Figueroa CA. A mathematical model of coronary blood flow control: simulation of patient-specific three-dimensional hemodynamics during exercise. Am J Physiol Circ Physiol 310: H1242-H1258, 2016. doi: 10.1152/ajpheart.00517.2015.

7. Arts T, Reneman RS. Interaction Between Intramyocardial Pressure (IMP) and Myocardial Circulation. J Biomech Eng 107: 51, 1985. doi: 10.1115/1.3138520.

8. Ashikawa K, Kanatsuka H, Suzuki T, Takishima T. Phasic blood flow velocity pattern in epimyocardial microvessels in the beating canine left ventricle. Circ Res 59: 704-711, 1986. doi: 10.1161/01.RES.59.6.704.

9. Baek S, Rajagopal KR, Humphrey JD. A Theoretical Model of Enlarging Intracranial Fusiform Aneurysms. J Biomech Eng 128: 142, 2006. doi: 10.1115/1.2132374.

10. Baek S, Valentín A, Humphrey JD. Biochemomechanics of Cerebral Vasospasm and its Resolution: II. Constitutive Relations and Model Simulations. Ann Biomed Eng 35: 1498, 2007. doi: 10.1007/s10439-007-9322-x.

11. Ball RM, Bache RJ. Distribution of myocardial blood flow in the exercising dog with restricted coronary artery inflow. [Online]. Circ Res 38: 60-6, 1976. http://www.ncbi.nlm.nih.gov/pubmed/1245021 [4 Apr. 2019].

12. Beard DA, Bassingthwaighte JB. The Fractal Nature of Myocardial Blood Flow Emerges from a Whole-Organ Model of Arterial Network. J Vasc Res 37: 282-296, 2000. doi: 10.1159/000025742.

13. Camici PG, Crea F. Coronary Microvascular Dysfunction. N Engl J Med 356: 830-840, 2007. doi: 10.1056/NEJMra061889.

14. Canty JM. Coronary pressure-function and steady-state pressure-flow relations during autoregulation in the unanesthetized dog. Circ Res 63: 821-836, 1988. doi: 10.1161/01.RES.63.4.821.

15. Carlson BE, Arciero JC, Secomb TW. Theoretical model of blood flow autoregulation: roles of myogenic, shear-dependent, and metabolic responses. Am J Physiol Circ Physiol 295: H1572- 
H1579, 2008. doi: 10.1152/ajpheart.00262.2008.

16. Chilian WM, Layne SM. Coronary microvascular responses to reductions in perfusion pressure. Evidence for persistent arteriolar vasomotor tone during coronary hypoperfusion. Circ Res 66: 1227-1238, 1990. doi: 10.1161/01.RES.66.5.1227.

17. Clifford PS, Kluess HA, Hamann JJ, Buckwalter JB, Jasperse JL. Mechanical compression elicits vasodilatation in rat skeletal muscle feed arteries. J Physiol 572: 561-567, 2006. doi: 10.1113/jphysiol.2005.099507.

18. Cornelissen AJM, Dankelman J, VanBavel E, Spaan JAE. Balance between myogenic, flowdependent, and metabolic flow control in coronary arterial tree: a model study. Am J Physiol Circ Physiol 282: H2224-H2237, 2002. doi: 10.1152/ajpheart.00491.2001.

19. Dankelman J, Vergroesen I, Han Y, Spaan JA. Dynamic response of coronary regulation to heart rate and perfusion changes in dogs. Am J Physiol 263: H447-52, 1992. doi: 10.1152/ajpheart.1992.263.2.H447.

20. De Waard GA, Cook CM, Van Royen N, Davies JE. Coronary autoregulation and assessment of stenosis severity without pharmacological vasodilation. Eur Heart J 39: 4062-4071, 2018. doi: 10.1093/eurheartj/ehx669.

21. Dick GM, Namani R, Patel B, Kassab GS. Role of Coronary Myogenic Response in Pressure-Flow Autoregulation in Swine: A Meta-Analysis With Coronary Flow Modeling. Front Physiol 9: 580, 2018. doi: 10.3389/fphys.2018.00580.

22. Duncker DJ, Bache RJ. Regulation of Coronary Blood Flow During Exercise. Physiol Rev 88: 10091086, 2008. doi: 10.1152/physrev.00045.2006.

23. Duncker DJ, Koller A, Merkus D, Canty JM. Regulation of Coronary Blood Flow in Health and Ischemic Heart Disease. Prog Cardiovasc Dis 57: 409-422, 2015. doi: 10.1016/J.PCAD.2014.12.002.

24. Farias $\mathbf{M}$, Gorman MW, Savage $\mathbf{M}$ V., Feigl EO. Plasma ATP during exercise: possible role in regulation of coronary blood flow. Am J Physiol Circ Physiol 288: H1586-H1590, 2005. doi: 10.1152/ajpheart.00983.2004.

25. Feigl EO. Coronary physiology. Physiol Rev 63: 1-205, 1983. doi: 10.1152/physrev.1983.63.1.1.

26. Ferruzzi J, Vorp DA, Humphrey JD. On constitutive descriptors of the biaxial mechanical behaviour of human abdominal aorta and aneurysms. J R Soc Interface 8: 435-450, 2011. doi: 10.1098/rsif.2010.0299.

27. Fibich G, Lanir Y, Liron N. Mathematical model of blood flow in a coronary capillary. Am J Physiol - Hear Circ Physiol 265, 1993. doi: 10.1152/ajpheart.1993.265.5.h1829.

28. Figueroa CA, Baek S, Taylor CA, Humphrey JD. A computational framework for fluid-solidgrowth modeling in cardiovascular simulations. Comput Methods Appl Mech Eng 198: 35833602, 2009. doi: 10.1016/j.cma.2008.09.013.

29. Filonova V, Gharahi H, Nama N, Baek S, Figueroa CA. A Multiscale Framework for Defining Homeostasis in Distal Vascular Trees: Applications to the Pulmonary Circulation [Online]. 2020. http://arxiv.org/abs/2001.04880. 
30. Gleason RL, Taber LA, Humphrey JD. A 2-D model of flow-induced alterations in the geometry, structure, and properties of carotid arteries. J Biomech Eng 126: 371-381, 2004. doi: 10.1115/1.1762899.

31. Goodwill AG, Dick GM, Kiel AM, Tune JD. Regulation of Coronary Blood Flow. In: Comprehensive Physiology. John Wiley \& Sons, Inc., p. 321-382.

32. Grände $\mathbf{P}$, Borgström $\mathbf{P}$, Mellander $\mathbf{S}$. On the nature of basal vascular tone in cat skeletal muscle and its dependence on transmural pressure stimuli. Acta Physiol Scand 107: 365-376, 1979. doi: 10.1111/j.1748-1716.1979.tb06487.x.

33. Guo X, Kassab GS. Distribution of stress and strain along the porcine aorta and coronary arterial tree. Am J Physiol Circ Physiol 286: H2361-H2368, 2004. doi: 10.1152/ajpheart.01079.2003.

34. Hayenga HN, Hu JJ, Meyer CA, Wilson E, Hein TW, Kuo L, Humphrey JD. Differential progressive remodeling of coronary and cerebral arteries and arterioles in an aortic coarctation model of hypertension. Front Physiol 3 NOV, 2012. doi: 10.3389/fphys.2012.00420.

35. Hoffman JI, Spaan JA. Pressure-flow relations in coronary circulation. Physiol Rev 70: 331-390, 1990. doi: 10.1152/physrev.1990.70.2.331.

36. Humphrey JD. Cardiovascular Solid Mechanics: Cells, Tissues, and Organs [Online]. SpringerVerlag. file://www.springer.com/us/book/9780387951683.

37. HUMPHREY JD, RAJAGOPAL KR. A CONSTRAINED MIXTURE MODEL FOR GROWTH AND REMODELING OF SOFT TISSUES. Math Model Methods App/ Sci 12: 407-430, 2002. doi: $10.1142 / \mathrm{S} 0218202502001714$.

38. Jacobsen JCB, Mulvany MJ, Holstein-Rathlou NH. A mechanism for arteriolar remodeling based on maintenance of smooth muscle cell activation. Am J Physiol - Regul Integr Comp Physiol 294: R1379-R1389, 2008. doi: 10.1152/ajpregu.00407.2007.

39. Jaquet C, Najman L, Talbot H, Grady U, Schaap M, Spain B, Kim HJ, Vignon-Clementel I, Taylor CA. Generation of patient-specific cardiac vascular networks: a hybrid image-based and synthetic geometric model. .

40. Johnson PC. The Myogenic Response. In: Comprehensive Physiology. John Wiley \& Sons, Inc., p. 409-442.

41. Jones CJH, Kuo L, Davis MJ, Defily D V., Chilian WM. Role of Nitric Oxide in the Coronary Microvascular Responses to Adenosine and Increased Metabolic Demand. Circulation 91: 18071813, 1995. doi: 10.1161/01.CIR.91.6.1807.

42. Kaimovitz B, Lanir Y, Kassab GS. Large-Scale 3-D Geometric Reconstruction of the Porcine Coronary Arterial Vasculature Based on Detailed Anatomical Data. Ann Biomed Eng 33: 15171535, 2005. doi: 10.1007/s10439-005-7544-3.

43. Kaimovitz B, Lanir Y, Kassab GS. A full 3-D reconstruction of the entire porcine coronary vasculature. Am J Physiol Circ Physiol 299: H1064-H1076, 2010. doi: 10.1152/ajpheart.00151.2010.

44. Kanatsuka H, Lamping KG, Eastham CL, Dellsperger KC, Marcus ML. Comparison of the effects of increased myocardial oxygen consumption and adenosine on the coronary microvascular 
resistance. Circ Res 65: 1296-1305, 1989. doi: 10.1161/01.RES.65.5.1296.

45. Kanatsuka H, Lamping KG, Eastham CL, Marcus ML. Heterogeneous changes in epimyocardial microvascular size during graded coronary stenosis. Evidence of the microvascular site for autoregulation. [Online]. Circ Res 66: 389-96, 1990.

http://www.ncbi.nlm.nih.gov/pubmed/2297810 [21 Feb. 2019].

46. Karch R, Neumann F, Neumann M, Schreiner W. Staged Growth of Optimized Arterial Model Trees. Ann Biomed Eng 28: 495-511, 2000. doi: 10.1114/1.290.

47. Kassab GS, Fung YC. The pattern of coronary arteriolar bifurcations and the uniform shear hypothesis [Online]. Ann Biomed Eng 23: 13-20, 1995.

http://www.ncbi.nlm.nih.gov/pubmed/7762878.

48. Kassab GS, Rider CA, Tang NJ, Fung YC. Morphometry of pig coronary arterial trees. Am J Physiol Circ Physiol 265: H350-H365, 1993. doi: 10.1152/ajpheart.1993.265.1.H350.

49. Kiel AM, Goodwill AG, Baker HE, Dick GM, Tune JD. Local metabolic hypothesis is not sufficient to explain coronary autoregulatory behavior. Basic Res Cardiol 113: 33, 2018. doi: 10.1007/s00395-018-0691-0.

50. Kirk ES, Honig CR. An experimental and theoretical analysis of myocardial tissue pressure. Am J Physiol Content 207: 361-367, 1964. doi: 10.1152/ajplegacy.1964.207.2.361.

51. Kopincová J, Púzserová A, Bernátová I. L-NAME in the cardiovascular system - Nitric oxide synthase activator? Pharmacol. Reports 64 Elsevier B.V.: 511-520, 2012.

52. Kuo L, Chilian WM, Davis MJ. Coronary arteriolar myogenic response is independent of endothelium. Circ Res 66: 860-866, 1990. doi: 10.1161/01.RES.66.3.860.

53. Kuo L, Davis MJ, Chilian WM. Myogenic activity in isolated subepicardial and subendocardial coronary arterioles. Am J Physiol 255: H1558-62, 1988. doi: 10.1152/ajpheart.1988.255.6.H1558.

54. Kuo L, Davis MJ, Chilian WM. Longitudinal Gradients for Endothelium-Dependent and Independent Vascular Responses in the Coronary Microcirculation. Circulation 92: 518-525, 1995. doi: 10.1161/01.CIR.92.3.518.

55. Liao JC, Kuo L. Interaction between adenosine and flow-induced dilation in coronary microvascular network. Am J Physiol 272: H1571-81, 1997. doi:

10.1152/ajpheart.1997.272.4.H1571.

56. Lindström SB, Satha G, Klarbring A. Extension of Murray's law including nonlinear mechanics of a composite artery wall. Biomech Model Mechanobiol 14: 83-91, 2015. doi: 10.1007/s10237-0140590-8.

57. Liu D, Wood NB, Witt N, Hughes AD, Thom SA, Xu XY. Assessment of Energy Requirement for the Retinal Arterial Network in Normal and Hypertensive Subjects. J Biomech Eng 134: 14501-14507, 2012. doi: 10.1115/1.4005529.

58. Martinez-Lemus LA. The dynamic structure of arterioles. Basic Clin Pharmacol Toxicol 110: 5-11, 2012. doi: 10.1111/j.1742-7843.2011.00813.x.

59. Martinez-Lemus LA, Wu X, Wilson E, Hill MA, Davis GE, Davis MJ, Meininger GA. Integrins as 
Unique Receptors for Vascular Control. J Vasc Res 40: 211-233, 2003. doi: 10.1159/000071886.

60. Mayrovitz HN, Roy J. Microvascular blood flow: evidence indicating a cubic dependence on arteriolar diameter. Am J Physiol Circ Physiol 245: H1031-H1038, 1983. doi: 10.1152/ajpheart.1983.245.6.H1031.

61. Meininger GA, Mack CA, Fehr KL, Bohlen HG. Myogenic vasoregulation overrides local metabolic control in resting rat skeletal muscle. [Online]. Circ Res 60: 861-70, 1987. http://www.ncbi.nlm.nih.gov/pubmed/3594758 [3 Apr. 2019].

62. Mittal N, Zhou Y, Linares C, Ung S, Kaimovitz B, Molloi S, Kassab GS. Analysis of blood flow in the entire coronary arterial tree. Am J Physiol Circ Physiol 289: H439-H446, 2005. doi: 10.1152/ajpheart.00730.2004.

63. Murray CD. The physiological principle of minimum work applied to the angle of branching of arteries. Proc Natl Acad Sci 12: 835-841, 1926.

64. Murray CD. The Physiological Principle of Minimum Work [Online]. Proc Natl Acad Sci U S A 12: 207-214, 1926. https://www.ncbi.nlm.nih.gov/pmc/articles/PMC1084489/.

65. Mynard JP, Smolich JJ. Influence of anatomical dominance and hypertension on coronary conduit arterial and microcirculatory flow patterns: a multiscale modeling study. Am J Physiol Circ Physiol 311: H11-H23, 2016. doi: 10.1152/ajpheart.00997.2015.

66. Namani R, Kassab GS, Lanir Y. Integrative model of coronary flow in anatomically based vasculature under myogenic, shear, and metabolic regulation. J Gen Physiol 150: 145-168, 2018. doi: 10.1085/JGP.201711795.

67. Namani R, Kassab GS, Lanir Y. Morphometric Reconstruction of Coronary Vasculature Incorporating Uniformity of Flow Dispersion. Front Physiol 9: 1069, 2018. doi: 10.3389/fphys.2018.01069.

68. Paul R. Chemical energetics of vascular smooth muscle. Compr Physiol 2007: 1-16, 1980. doi: 10.1002/cphy.cp020209.

69. Pradhan RK, Feigl EO, Gorman MW, Brengelmann GL, Beard DA. Open-loop (feed-forward) and feedback control of coronary blood flow during exercise, cardiac pacing, and pressure changes. Am J Physiol Circ Physiol 310: H1683-H1694, 2016. doi: 10.1152/ajpheart.00663.2015.

70. Pries AR, Badimon L, Bugiardini R, Camici PG, Dorobantu M, Duncker DJ, Escaned J, Koller A, Piek JJ, de Wit C. Coronary vascular regulation, remodelling, and collateralization: mechanisms and clinical implications on behalf of the working group on coronary pathophysiology and microcirculation. Eur Heart J 36: 3134-3146, 2015. doi: 10.1093/eurheartj/ehv100.

71. Pries AR, Reglin B, Secomb TW. Remodeling of Blood Vessels. Hypertension 46: 725-731, 2005. doi: 10.1161/01.HYP.0000184428.16429.be.

72. Pries AR, Secomb TW, Gaehtgens P. Design Principles of Vascular Beds. Circ Res 77: 1017-1023, 1995. doi: 10.1161/01.RES.77.5.1017.

73. Pries AR, Secomb TW, Gessner T, Sperandio MB, Gross JF, Gaehtgens P. Resistance to blood flow in microvessels in vivo. Circ Res 75: 904-915, 1994. doi: 10.1161/01.RES.75.5.904. 
74. Reesink KD, Spronck B. Constitutive interpretation of arterial stiffness in clinical studies: A methodological review. Am J Physiol - Hear Circ Physiol 316: H693-H709, 2019. doi: 10.1152/ajpheart.00388.2018.

75. Rhodin JAG. The ultrastructure of mammalian arterioles and precapillary sphincters. $J$ Ultrastruct Res 18: 181-223, 1967. doi: 10.1016/S0022-5320(67)80239-9.

76. Seyedsalehi S, Zhang L, Choi J, Baek S. Prior Distributions of Material Parameters for Bayesian Calibration of Growth and Remodeling Computational Model of Abdominal Aortic Wall. J Biomech Eng 137: 101001, 2015. doi: 10.1115/1.4031116.

77. Smith NP, Pullan AJ, Hunter PJ. Generation of an Anatomically Based Geometric Coronary Model. Ann Biomed Eng 28: 14-25, 2000. doi: 10.1114/1.250.

78. Stepp DW, Nishikawa Y, Chilian WM. Regulation of Shear Stress in the Canine Coronary Microcirculation [Online]. http://www.circulationaha.org [11 Mar. 2019].

79. Suwa N, Niwa T, Fukasawa H, Sasaki Y. Estimation of Intravascular Blood Pressure Gradient by Mathematical Analysis of Arterial Casts. Tohoku J Exp Med 79: 168-198, 1963. doi: 10.1620/tjem.79.168.

80. Taber LA. An optimization principle for vascular radius including the effects of smooth muscle tone. [Online]. Biophys J 74: 109-114, 1998. https://www.ncbi.nlm.nih.gov/pmc/articles/PMC1299367/.

81. Tiefenbacher CP, Chilian WM. Heterogeneity of coronary vasomotion. [Online]. Basic Res Cardiol 93: 446-54, 1998. http://www.ncbi.nlm.nih.gov/pubmed/9879450 [5 Mar. 2019].

82. Tillmanns H, Ikeda S, Hansen H, Sarma JS, Fauvel JM, Bing RJ. Microcirculation in the ventricle of the dog and turtle. [Online]. Circ Res 34: 561-9, 1974.

http://www.ncbi.nlm.nih.gov/pubmed/4826931 [21 Mar. 2019].

83. Trask AJ, Katz PS, Kelly AP, Galantowicz ML, Cismowski MJ, West TA, Neeb ZP, Berwick ZC, Goodwill AG, Alloosh M, Tune JD, Sturek M, Lucchesi PA. Dynamic micro- and macrovascular remodeling in coronary circulation of obese Ossabaw pigs with metabolic syndrome. J Appl Physiol 113: 1128-1140, 2012. doi: 10.1152/japplphysiol.00604.2012.

84. Valentín A, Humphrey JD. Parameter sensitivity study of a constrained mixture model of arterial growth and remodeling. J Biomech Eng 131, 2009. doi: 10.1115/1.3192144.

85. van den Akker J, Schoorl MJC, Bakker ENTP, vanBavel E. Small Artery Remodeling: Current Concepts and Questions. J Vasc Res 47: 183-202, 2010. doi: 10.1159/000255962.

86. VanBavel E, Spaan JA. Branching patterns in the porcine coronary arterial tree. Estimation of flow heterogeneity. [Online]. Circ Res 71: 1200-12, 1992.

http://www.ncbi.nlm.nih.gov/pubmed/1394880 [21 Mar. 2019].

87. VanBavel E, Tuna BG. Integrative Modeling of Small Artery Structure and Function Uncovers Critical Parameters for Diameter Regulation. PLoS One 9: e86901, 2014. doi: 10.1371/journal.pone.0086901.

88. Wolinsky H, Glagov S. Structural Basis for the Static Mechanical Properties of the Aortic. Circ Res 14: 400-413, 1964. doi: 10.1161/01.RES.14.5.400. 
89. Xie X, Wang Y. Flow Regulation in Coronary Vascular Tree: A Model Study. PLoS One 10: e0125778, 2015. doi: 10.1371/journal.pone.0125778.

90. Zeinali-Davarani S, Raguin LG, Vorp DA, Baek S. Identification of in vivo material and geometric parameters of a human aorta: toward patient-specific modeling of abdominal aortic aneurysm. Biomech Model Mechanobiol 10: 689-699, 2011. doi: 10.1007/s10237-010-0266-y.

91. Zeinali-Davarani S, Sheidaei A, Baek S. A finite element model of stress-mediated vascular adaptation: application to abdominal aortic aneurysms. Comput Methods Biomech Biomed Engin 14: 803-817, 2011. doi: 10.1080/10255842.2010.495344.

92. Zhang C, A Rogers P, Merkus D, M Muller-Delp J, P Tiefenbacher C, Potter B, D Knudson J, Rocic P, M Chilian W. Regulation of Coronary Microvascular Resistance in Health and Disease. . 